UCRL-ID-131536

NIF-10448-0A

\title{
NIF Title III Engineering Plan
}

\section{June 1998}

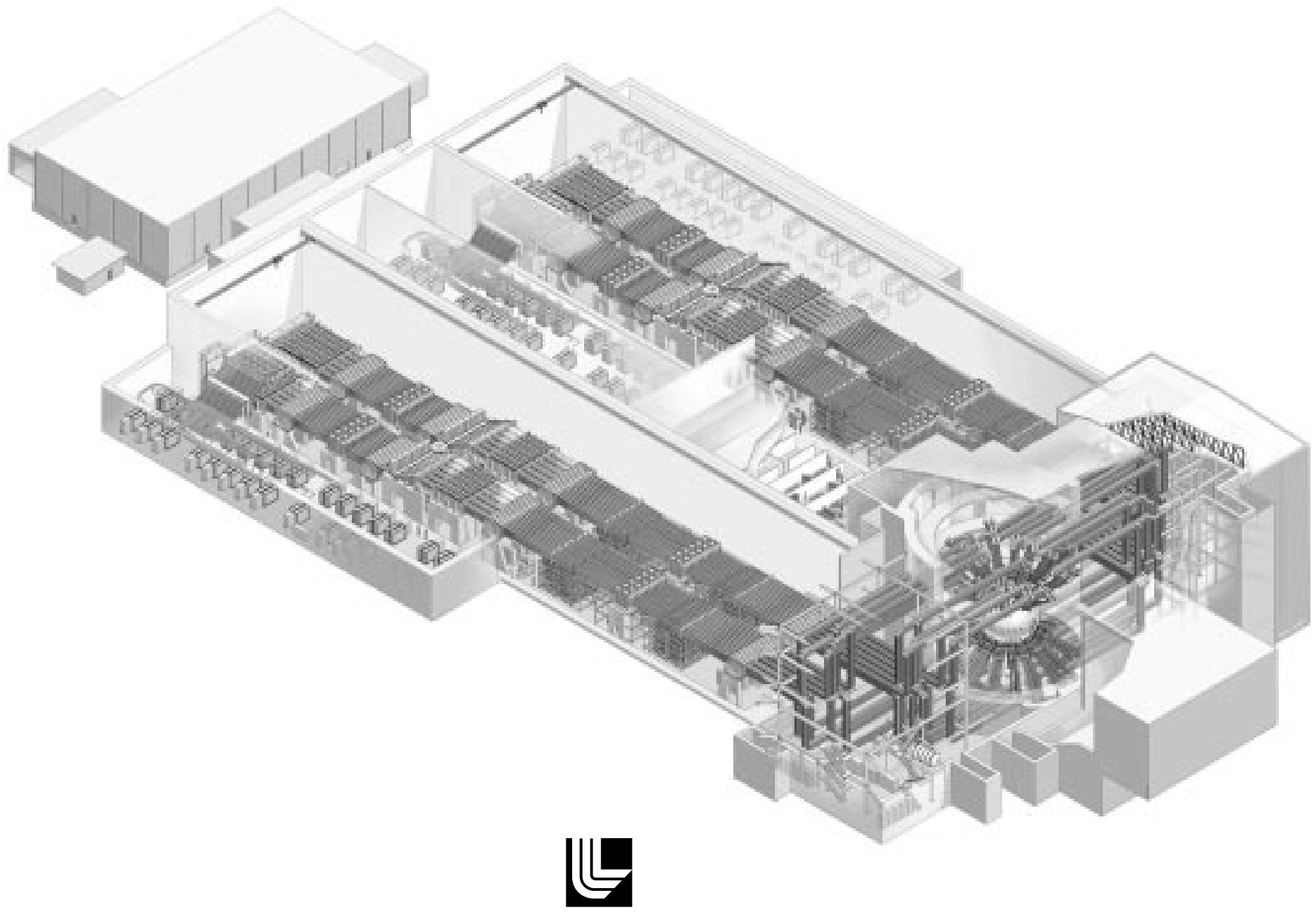


UCRL-ID-131536

\section{DISCLAIMER}

This document was prepared as an account of work sponsored by an agency of the United States Government. Neither the United States Government nor the University of California nor any of their employees, makes any warranty, express or implied, or assumes any legal liability or responsibility for the accuracy, completeness, or usefulness of any information, apparatus, product, or process disclosed, or represents that its use would not infringe privately owned rights. Reference herein to any specific commercial product, process, or service by trade name, trademark, manufacturer, or otherwise, does not necessarily constitute or imply its endorsement, recommendation, or favoring by the United States Government or the University of California. The views and opinions of authors expressed herein do not necessarily state or reflect those of the United States Government or the University of California, and shall not be used for advertising or product endorsement purposes.

This report has been reproduced directly from the best available copy.

Available to DOE and DOE contractors from the Office of Scientific and Technical Information

P.O. Box 62, Oak Ridge, TN 37831

Prices available from (615) 576-8401, FTS 626-8401

Available to the public from the

National Technical Information Service

U.S. Department of Commerce

5285 Port Royal Rd.,

Springfield, VA 22161

Work performed under the auspices of the U.S. Department of Energy by Lawrence Livermore National Laboratory under Contract W-7405-ENG-48. 


\section{NIF Title III \\ Engineering Plan}

June 1998 


\section{NIF Title III Engineering Plan}

\begin{tabular}{|c|c|c|}
\hline & & $\begin{array}{l}\text { Date approved } \\
\text { in NIF PDMS }\end{array}$ \\
\hline $\begin{array}{l}\text { Issued by: } \\
\text { J. Paisner }\end{array}$ & Project Manager & \\
\hline $\begin{array}{l}\text { Concurrance: } \\
\text { R. Sawicki }\end{array}$ & APE for Special Equipment & $7 / 13 / 98$ \\
\hline D. Larson & Laser System Engineer & $7 / 17 / 98$ \\
\hline J. Bowers & Beam Transport System Engineer & $7 / 13 / 98$ \\
\hline R. English & Optomechanical System Engineer & $7 / 15 / 98$ \\
\hline E. Bliss & System Control System Engineer & $7 / 10 / 98$ \\
\hline P. VanArsdall & $\begin{array}{l}\text { Deputy System Engineer for Integrated Computer } \\
\text { Control System }\end{array}$ & $7 / 13 / 98$ \\
\hline D. Atkinson & Target Area System Engineer & $7 / 17 / 98$ \\
\hline J. Atherton & APL for Optics & $7 / 17 / 98$ \\
\hline C. Belser & System Engineer for Optics & $7 / 17 / 98$ \\
\hline C. Clower & $\begin{array}{l}\text { APE for Start-up, Performance Testing, and } \\
\text { Operations }\end{array}$ & $7 / 17 / 98$ \\
\hline M. Lane & $\begin{array}{l}\text { Deputy APE for Start-up, Performance Testing, } \\
\text { and Operations }\end{array}$ & $7 / 17 / 98$ \\
\hline E. Grasz & Transport and Handling System Engineer & $7 / 15 / 98$ \\
\hline O. Nazario & Information Systems System Engineer & \\
\hline G. Kugler & $\begin{array}{l}\text { Conventional Facilities Title III Engineering } \\
\text { Manager }\end{array}$ & $7 / 17 / 98$ \\
\hline P. Kempel & System Engineer for Conventional Facility Design & $7 / 17 / 98$ \\
\hline V. Roberts & Conventional Facility Construction Manager & $7 / 17 / 98$ \\
\hline J. Yatabe & Assurances Manager & $7 / 9 / 98$ \\
\hline R. Wolfe & QA Manager & $7 / 15 / 98$ \\
\hline $\begin{array}{l}\text { Prepared by: } \\
\text { G. Deis }\end{array}$ & $\begin{array}{l}\text { Systems Integration and } \\
\text { Engineering Services Manager }\end{array}$ & $7 / 8 / 98$ \\
\hline
\end{tabular}




\section{Contents}

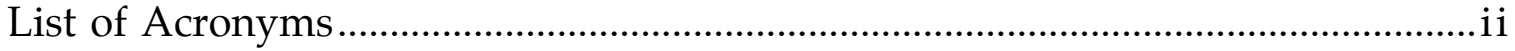

List of Figures and Tables.........................................................................................ii

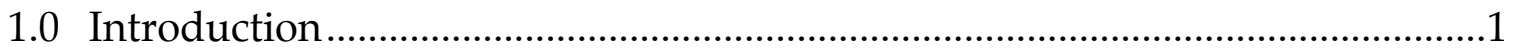

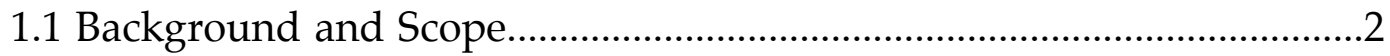

1.2 Purpose of this Document.........................................................................

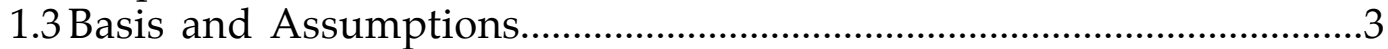

1.4 Work Breakdown Structure ……………….............................................

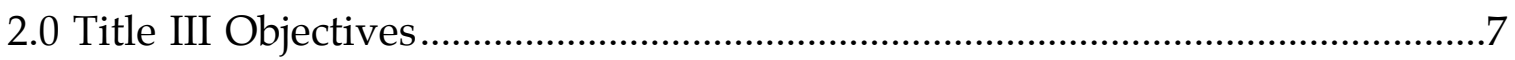

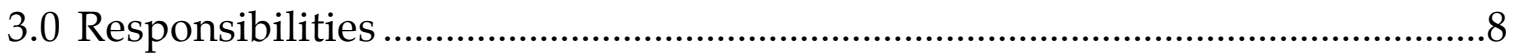

4.0 Title III Deliverables, Responsibilities, and Acceptance Criteria.....................10

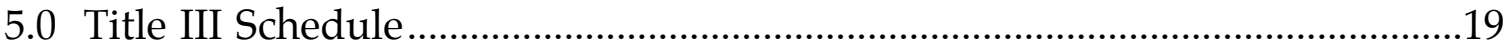

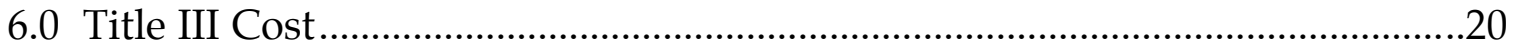

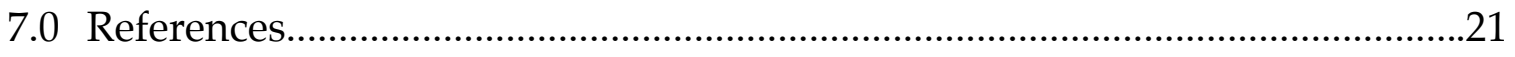

Appendix A - Glossary of Important Definitions ...................................................23

Appendix B - Summary of Title III Deliverables ...................................................25

Appendix C - Sample Format and Content for Deliverables ................................26

Appendix C-1 - Sample format and content for As-Built Bills of

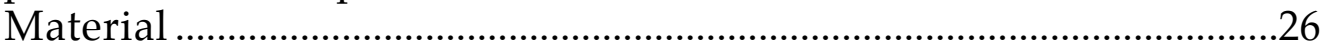

Appendix C-2 - Sample format and content for As-Built Dwgs, specifications, etc. ………………….......................................................27

Appendix C-3 - Sample format and content for Final Subsystem Completion Checklist..................................................................................28

Appendix C-4 - Sample format and content for Part/Component History Files by Serial Number................................................................30

Appendix C-5 - Title III Engineering Basis Book Outline ............................31

Appendix D - Revision Record ............................................................................33 


\section{List of Acronyms}

NIF National Ignition Facility

DOE US Department of Energy

AE Architect Engineer Firm

$\mathrm{CM}$ Construction Management firm

PC Primary Criteria

FR Functional Requirement

SDR System Design Requirement

SSDR Sub-System Design Requirement

ICF Inertial Confinement Fusion

ORR Operational Readiness Review

NEPA National Environmental Policy Act

WBS Work Breakdown Structure

FSAR Final Safety Analysis Report

APE Associate Project Engineer

IPS Integrated Project Schedule

ERP Enterprise Resource Planning (System)

LRU Line Replaceable Unit 


\section{List of Figures and Tables}

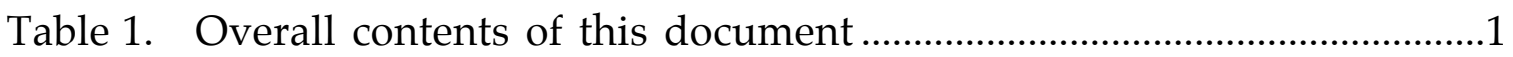

Figure 1. NIF WBS tree.....................................................................................6

Figure 2. Information handoff during Title III Engineering .................................

Table 2. Description of Title III deliverables..........................................................11 


\subsection{Introduction}

This National Ignition Facility (NIF) Title III Engineering Plan defines the engineering work to be performed by the NIF Project Team, in parallel with hardware procurement, fabrication, assembly, and installation, between the end of Title II design and the completion of the Project. This period is from mid-FY1998 to FY2003, with the beginning of Title III engineering being phased by subsystem according to the requirements of the integrated project schedule.

This plan includes seven sections and four appendices. The purpose of each section and Appendix is outlined in Table 1.

Table 1 - Overall Contents of this document

\begin{tabular}{|c|l|l|}
\hline Section & \multicolumn{1}{|c|}{ Title } & \multicolumn{1}{|c|}{ Purpose } \\
\hline 1 & Introduction & $\begin{array}{l}\text { General introduction, background, } \\
\text { scope, and purpose of the } \\
\text { document }\end{array}$ \\
\hline 2 & Title III Objectives & Overall objectives of Title III \\
\hline 3 & Responsibilities & $\begin{array}{l}\text { Overall responsibilities of } \\
\text { participants in Title III }\end{array}$ \\
\hline 4 & $\begin{array}{l}\text { Title III Deliverables, } \\
\text { Acsponsibilities, and }\end{array}$ & $\begin{array}{l}\text { the heart of the document; a } \\
\text { detailed listing of Title III } \\
\text { deliverables, including } \\
\text { descriptions of each, detailed } \\
\text { responsibilities, and acceptance } \\
\text { criteria. }\end{array}$ \\
\hline 5 & Title III Schedule & $\begin{array}{l}\text { General comments on Title III } \\
\text { schedule }\end{array}$ \\
\hline 6 & Title III Cost & General comments on Title III cost \\
\hline 7 & References & Listing of reference documents \\
\hline App A & Glossary of Important Terms & $\begin{array}{l}\text { Ddefinition of important terms } \\
\text { used in this document }\end{array}$ \\
\hline App B & $\begin{array}{l}\text { Summary of Title III } \\
\text { deliverables }\end{array}$ & $\begin{array}{l}\text { A one-page summary listing of } \\
\text { Title III deliverables }\end{array}$ \\
\hline
\end{tabular}




\begin{tabular}{|c|l|l|}
\hline App C & $\begin{array}{l}\text { Sample Format and Content } \\
\text { for Deliverables }\end{array}$ & $\begin{array}{l}\text { Sample formats for Title III } \\
\text { dliverables }\end{array}$ \\
\hline App D & Revision Record & Record of release and revision \\
\hline
\end{tabular}

\subsection{Background and Scope}

Title I design, which was completed, reviewed, and approved in December 1996, included all work necessary to complete the preliminary design of the NIF Project and established an integrated definition of all major subsystems with associated cost and schedule projections for project completion. With the completion of Title I, the facility and special equipment designs were mature enough to justify the release of resources to proceed with final engineering design details. Title I activity ended with a formal design review and U.S. Department of Energy (DOE) approval to proceed to Title II design (i.e., detailed design).

Title II design ${ }^{1}$ included all work necessary to define the detailed design configuration of the Project, incorporating all the necessary details, and then to review, check, approve, and accept the documentation into the procurement and construction packages. It also included preparation of acceptance test requirements and Project cost estimates to support bid evaluation.

Title III engineering begins at the initiation of procurement action, i.e., the submission of requisitions. It extends from the beginning of the fabrication/construction phase and continues through the completion of construction and equipment acceptance tests. It includes field engineering, problem resolution, configuration control, documentation maintenance, and the technical staff required to maintain all engineering records, including asbuilt drawings at Project completion. It includes the revision of existing design documentation and the generation of any new design documentation required as construction proceeds, engineering analysis to evaluate proposed design changes, and engineering support for inspection, assembly, installation, and testing.

Title III engineering services for facilities and conventional equipment are provided under contract with an Architect Engineer firm (AE) working with a Construction Management firm (CM). Title III engineering for special equipment is furnished by the Laboratory Project Staff. 


\subsection{Purpose of this Document}

The purpose of this document is to define the work that must be accomplished by the NIF Project during Title III Engineering. This definition is intended to be sufficiently detailed to provide a framework for yearly planning, to clearly identify the specific deliverables so that the Project teams can focus on them, and to provide a common set of objectives and processes across the Project. This plan has been preceded by similar documents for Title $\mathrm{I}^{2}$ and Title $\mathrm{II}^{1}$ design and complements the Site Management Plan ${ }^{3}$, the Project Control Manual ${ }^{4}$, the Quality Assurance Program Plan ${ }^{5}$, the RM Parsons NIF Title III Configuration Control Plan ${ }^{6}$, the Integrated Project Schedule ${ }^{7}$, the Preliminary Safety Analysis Report ${ }^{8}$, the Configuration Management Plan ${ }^{9}$, and the Transition Plan ${ }^{10}$.

This plan is not intended to be a stand-alone document. The NIF Project has developed an extensive base of source documents for requirements, procedures, plans, etc. In addition, the technical, cost, and schedule baselines are formally managed separately from this plan. In order to avoid duplication of information contained in other documents and the changemanagement burden that would result, this plan makes extensive reference to other documents when those documents are the primary source of information. This document is meant as an overview and does not supercede more specific documents. The current revision of the referenced documents should be consulted for definitive information.

\subsection{Basis and Assumptions}

The basis for NIF Title III engineering is the Title II (detailed) design completed in a phased manner by subsystem, from March 1997 through August 2001. The design drawings, specifications, other procurement documentation, and governing design criteria (e.g., Primary Criteria (PC), Functional Requirements (FRs), System Design Requirements (SDRs), SubSystem Design Requirements (SSDRs), and Interface Control Documents (ICDs)) form the basis for Title III engineering. As a part of the Title II design phase, final design packages and procurement documents are formally reviewed, approved, and placed under configuration management.

The assumptions for this plan are as follows:

1. The project budgets for Title III activities are defined in the Cost Account Plans.

2. Title III begins October 1, 1998 and ends September 30, 2003. Individual subsystems begin Title III work earlier or later than this, depending on the requirements of the integrated project schedule. 
3. Project baseline change control will be achieved through a set of hierarchical Baseline Change Control Boards ${ }^{11}$.

4. Formal configuration management will be utilized within the project to maintain control of changes, traceability of designs, and control of costs.

5. A first laser "bundle" (consisting of 8 individual beamlines) will be deployed, started up, and tested in advance of all other bundles. Successive bundles will be started up sequentially, handed off to the Inertial Confinement Fusion (ICF) Program, and operated by the Program in parallel with the completion of other bundles.

6. An Operational Readiness Review (ORR) will be conducted after startup of the first bundle, and before handoff to the operating Program. Internal management prestart reviews will be conducted before startup of subsequent laser bundles.

7. The technical state at the end of the project is defined in Project Completion Criteria 12 .

8. No formal or informal design reviews will be commissioned by organizations outside the Project.

The participants in the Title III engineering and associated operatingfunded activities include DOE, Lawrence Livermore National Laboratory, Los Alamos National Laboratory, Sandia National Laboratories, University of Rochester's Laboratory for Laser Energetics, Argonne National Laboratory (National Environmental Policy Act (NEPA) document preparer), The Parsons Infrastructure and Technology Group, Inc., Albert C. Martin and Associates, Sverdrup Facilities, Inc., engineering support contractor(s), and other subcontractors for design and construction.. 


\subsection{Work Breakdown Structure}

The NIF Project Summary Work Breakdown Structure (WBS) shown in Figure 1 is the organizing element for all Title III Engineering activities ${ }^{13}$. The WBS organizes the technical work scopes, cost estimates and tracking, and integrated schedule. 


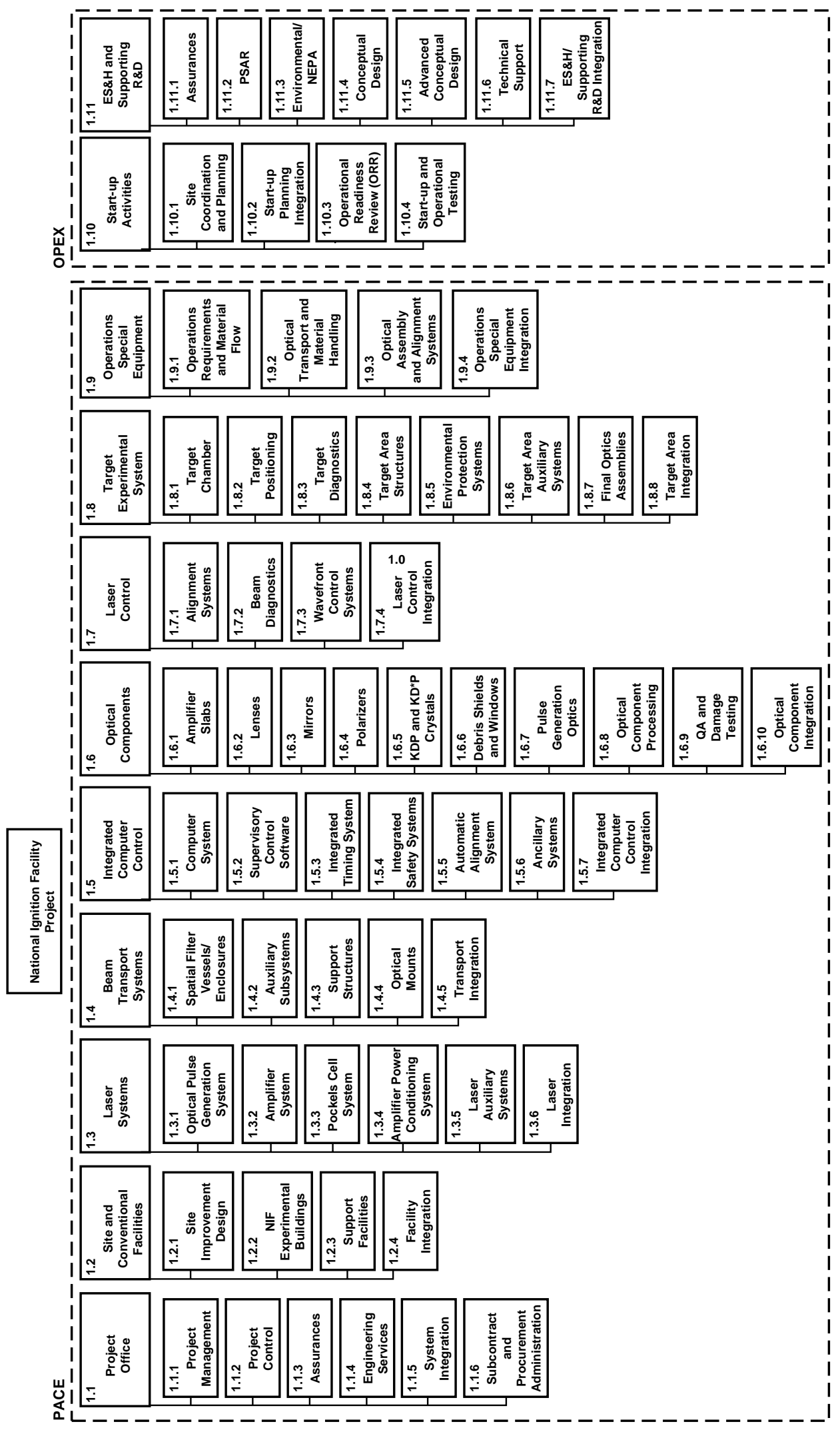

Figure 1. NIF WBS tree. 


\subsection{Title III Objectives}

The objective of Title III engineering is to support the procurement, fabrication, assembly, installation, and testing of NIF systems, structures, and components in a manner that achieves the completion of the NIF Project on schedule and within budget, meeting all technical requirements. As a part of completing the Project, Title III engineering will ensure that adequate information is developed, retained, and handed off to the ICF Program to permit safe and efficient operation.

The criteria of a successful Title III effort are:

- Deployment of high-quality hardware that achieves required operating parameters.

- Timely completion of project milestones.

- Controlled use of project contingency, and overall completion within budget.

- Accurately known as-built configuration.

- Smooth phased handoff to the ICF Program.

- Disciplined system-engineering approach to requirements verification and testing.

- Strict control of baseline changes.

- Successful Operational Readiness Review (ORR).

- Effective resolution of problems as they arise.

- Accurate and complete archival of project data.

- Successful completion of all externally-required documents (Final Safety Analysis Report (FSAR), etc).

These objectives form the basis for the deliverables outlined in Section 4 . Completion of those deliverables, in concert with completion of the hardware and software, will ensure the success of the NIF Project. 


\subsection{Responsibilities}

Responsibilities within the NIF Project are clearly defined.

- Delivery of subsystem hardware that meets technical requirements, onschedule and within budget, is the responsibility of the line organizations (i.e., those run by Associate Project Engineers (APEs)). This responsibility is delegated by the Project Manager to the Associate Project Engineers, the System Engineers, the Lead Engineers, their staff, and contractors. The Special Equipment and Optics responsibility is complete when the deployed hardware has been accepted by the NIF Start-up, Performance Testing, and Operations Group.

- Start-up and system activation is the responsibility of the NIF Start-up, Performance Testing, and Operations Group. It includes the acceptance of subsystem acceptance test results through the completion of bundleby-bundle operational tests and handoff to the ICF Program.

- Operation and maintenance of NIF systems is the responsibility of the ICF Program following bundle-by-bundle handoff from the NIF Startup, Performance Testing, and Operations Group.

- Smooth transition to operation is the joint responsibility of the NIF Project and ICF Program. Planning for this transition will be performed by an integrated team and approved by both organizations.

- Safety is the responsibility of the line organizations and each individual within the Project. The responsibilities are defined in the NIF Construction Safety Program ${ }^{14}$, the Conventional Facility Health and Safety Plan ${ }^{15}$, the NIF Startup/Operations Engineering and Special Equipment Construction Health and Safety Plan ${ }^{16}$, the Preliminary Safety Analysis Report ${ }^{8}$, the Final Safety Analysis Report (in preparation), and applicable Facility Safety Procedures and Operational Safety Procedures as they are developed.

- Configuration Management is the responsibility of the line organizations with overall leadership by the Configuration Management group (see Configuration Management Plan ${ }^{9}$ ). The process required (e.g., change control, change implementation) will be specified, administered, and supported by the Configuration Management group, and line organizations will use those processes to maintain accurate configuration accounting.

- Project Documentation is the responsibility of the line organizations. A significant number of Title III engineering deliverables relate to providing adequate documentation to the NIF Start-up, Performance Testing, and Operations team before subsystems can be approved as complete. 
- Quality Assurance is the responsibility of the line organizations. Line organizations will meet this responsibility by implementing the procedures in the Project Control Manual ${ }^{4}$ and the Quality Assurance Program Plan ${ }^{5}$. NIF Project Assurances will help line organizations achieve their quality goals by ensuring that the Project Control Manual contains appropriate requirements and guidance for performing quality-related activities. NIF Project Assurances will also assess implementation of these procedures.

- Construction Management is the responsibility of the Site Manager and Construction Managers, as described in the NIF Site Management Plan $^{3}$.

During Title III engineering, responsibility for hardware/software systems is handed off from the Special Equipment and Optics groups to the NIF Startup, Performance Testing, and Operations Group and ultimately to the Operating Program. Information of various types is also handed off from the Special Equipment and Optics Groups to the NIF Start-up, Performance Testing, and Operations Group and to the Operating Program (see Figure 2).

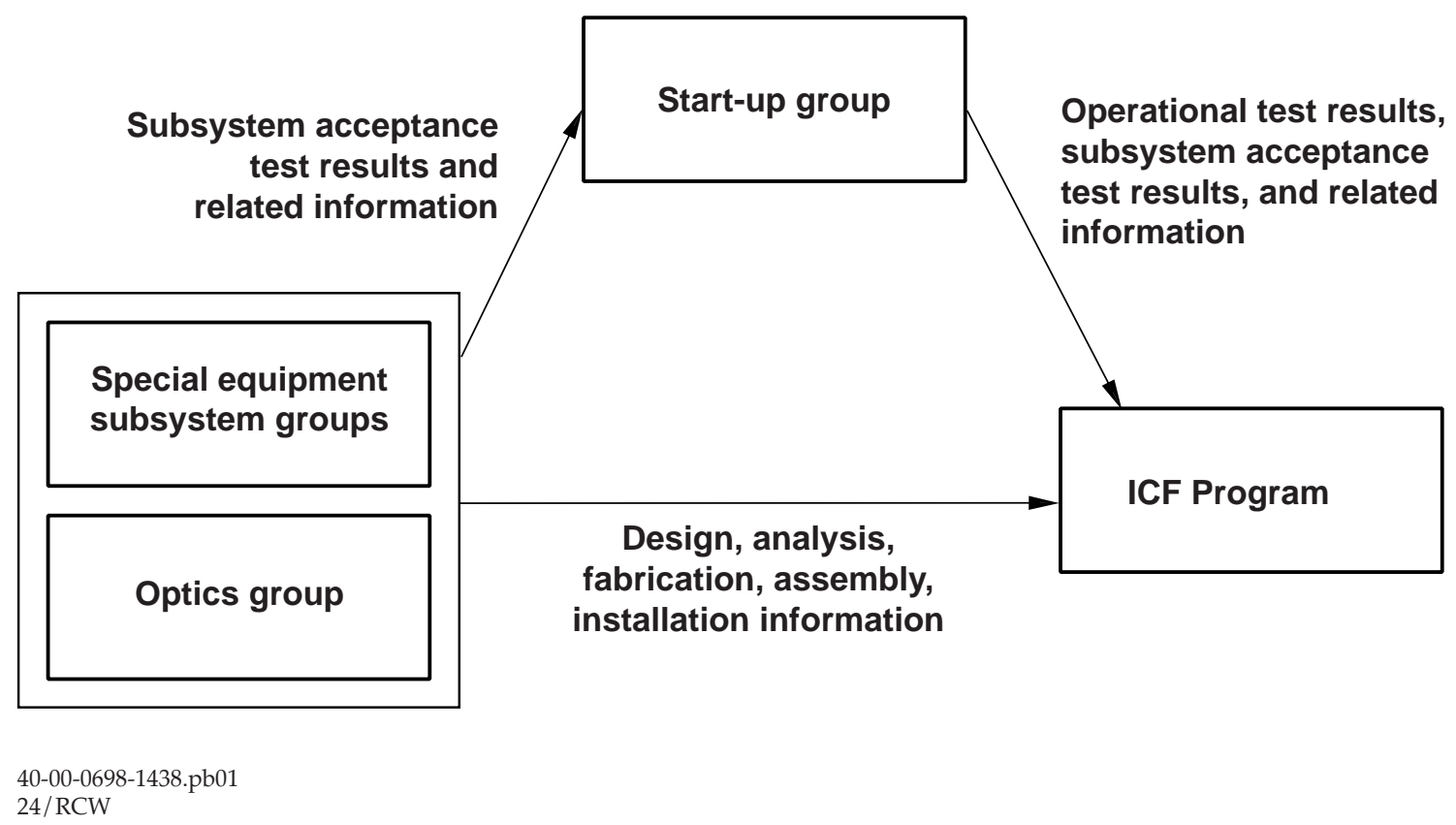

Figure 2. Information handoff during Title III Engineering.

Specific responsibility for deliverables is detailed in Section 4 . 


\subsection{Title III Deliverables, Responsibilities, and Acceptance Criteria}

Table 2 specifies the Title III deliverables.

The left 4 columns of this table identify each deliverable, its purpose, a description of the format and content, and the criteria that will be used to determine its acceptance. Appendices at the end of this document provide sample formats for many of the deliverables. This defines the "what" and the "why".

The fifth column indicates when it should be completed.

The next 12 columns specify which WBS elements shall provide each of the deliverables. An " $x$ " indicates the deliverable should be provided at WBS level 2. Numbers, such as ".1" and ".2" indicate which WBS level 3 areas are required to provide the deliverable. This is the first part of the definition of the "who".

The last 11 columns indicate the Project position title that is responsible for preparing, providing input, reviewing, approving, etc., each deliverable. This completes the definition of the "who". 
Table 2 - Description of Title III deliverables

\begin{tabular}{|c|c|c|c|c|c|c|c|c|c|c|c|c|c|c|c|c|c|c|c|c|c|c|c|c|c|c|}
\hline \multirow[b]{2}{*}{ Deliverable name } & \multirow[b]{2}{*}{ Purpose } & \multirow[b]{2}{*}{ Description } & \multirow[b]{2}{*}{ Acceptance Criteria } & \multirow[b]{2}{*}{ When Duee } & \multicolumn{11}{|c|}{ 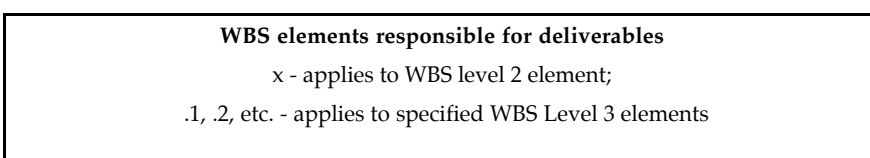 } & \multicolumn{11}{|c|}{\begin{tabular}{l|l} 
zuidance & $\begin{array}{c}\text { Responsible Individuals } \\
\text { I- Provides information } \\
\text { Prepares } \\
\text { ews }\end{array}$ C-concurs \\
ews
\end{tabular}} \\
\hline & & & & & 1.1 & 1.2 & 1.3 & 1.4 & 1.5 & 1.6 & 1.7 & 1.8 & 1.9 & 1.10 & 1.1 & 要 & 量量 & $\approx$ & & & 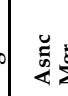 & & 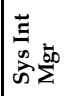 & 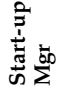 & 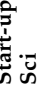 & Other \\
\hline $\begin{array}{l}\text { Project } \\
\text { Deliverables } \\
\text { (n) }\end{array}$ & & & & & & & & & & & & & & & & & & & & & & & & & & \\
\hline & 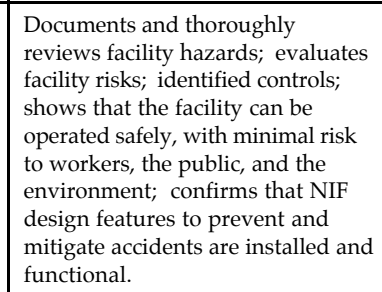 & 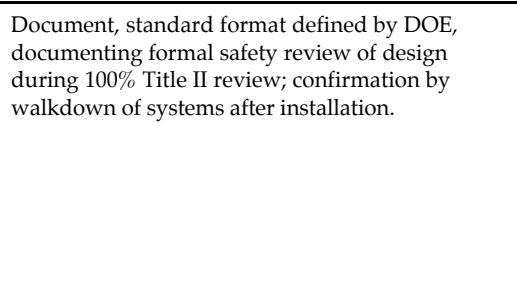 & 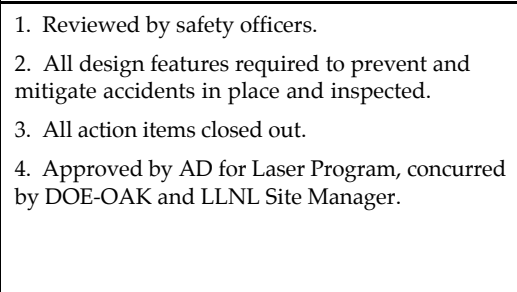 & by ORR & \begin{tabular}{c|}
$x$ \\
\end{tabular} & $x$ & $x$ & $x$ & $x^{x}$ & & $x$ & \begin{tabular}{|l|l}
$x$ \\
\end{tabular} & $x$ & $\begin{array}{ll}x \\
\end{array}$ & $x$ & $\mathrm{I}, \mathrm{R}$ & $\mathrm{I}, \mathrm{R}$ & $\mathrm{I}, \mathrm{s}$ & & c & $\mathrm{P}$ & & & & & 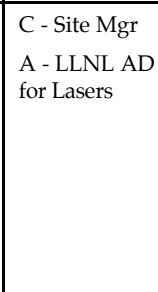 \\
\hline \begin{tabular}{l|} 
ORR for first bundle \\
\end{tabular} & 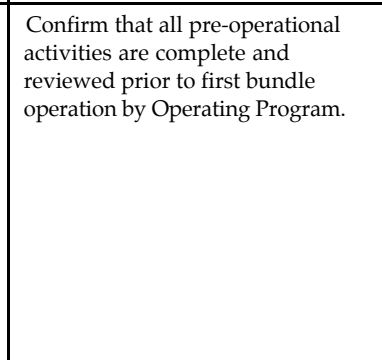 & 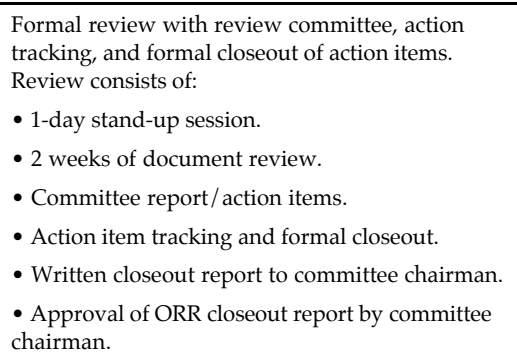 & 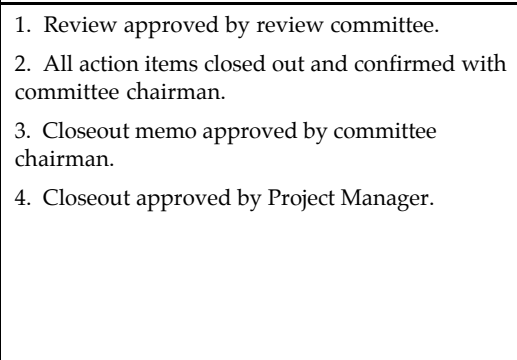 & 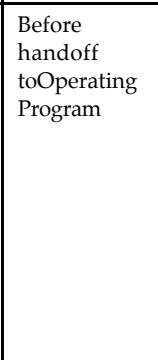 & & & & & & & & & & & & I & I & 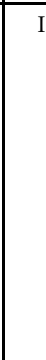 & & A & $\mathrm{C,G}$ & $\mathrm{R}$ & R & $\mathrm{P}$ & $\mathrm{R}$ & A-DOE \\
\hline 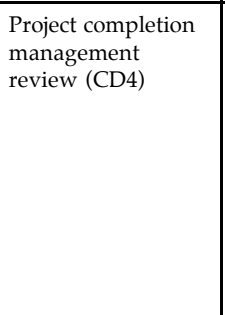 & 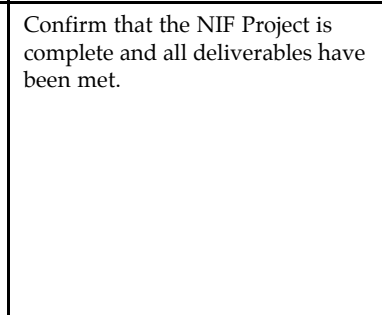 & 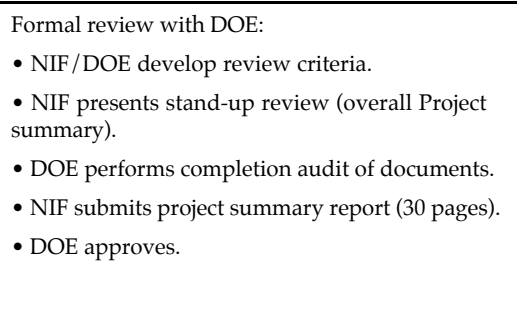 & 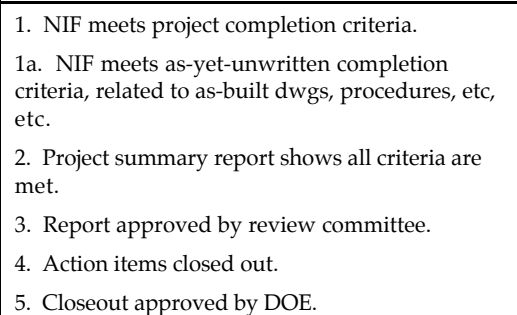 & 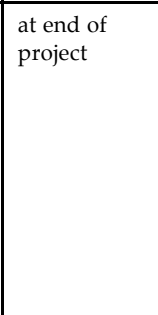 & $x^{x}$ & & & & & & & & & & & $\overline{1}$ & & ca & c & $\mathrm{P}$ & c & ${ }_{R}^{R}$ & $\begin{array}{ll}\text { R } \\
\end{array}$ & $\mathrm{R}^{\mathrm{R}}$ & R & A-DOE \\
\hline 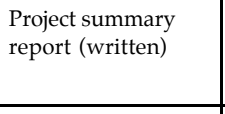 & $\begin{array}{l}\text { Document completion and } \\
\text { closeout of all project activities. }\end{array}$ & $\begin{array}{l}\text { Docoument, } 50 \text { pages, similar to SIS Program } \\
\text { dodurment }\end{array}$ & $\begin{array}{l}\text { 1. rivject summary report shows all criteria } \\
\text { are met. } \\
\text { 2. Reprt approved by project manager. }\end{array}$ & $\begin{array}{l}\text { before } \\
\text { conpletion } \\
\text { review }\end{array}$ & $x$ & & & & & & & & & & & & & F & $\mathrm{P}^{\mathrm{s}}$ & A & P & $\mathrm{P}^{\mathrm{p}}$ & P & ${ }^{\mathrm{P}}$ & & \\
\hline 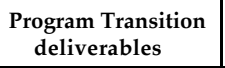 & & & & & & & & & & & & & & & & & & & & & & & & & & \\
\hline $\begin{array}{l}\begin{array}{l}\text { Procedures for } \\
\text { turnover to } \\
\text { operations }\end{array} \\
\end{array}$ & 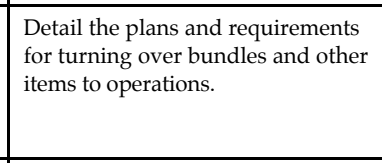 & 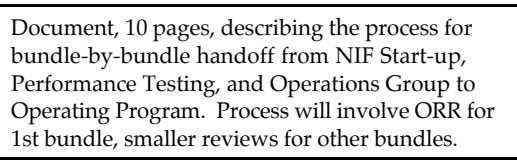 & 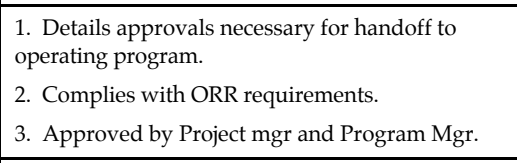 & \begin{tabular}{|l} 
early in Title \\
III TIt
\end{tabular} & & & & & & & & & & 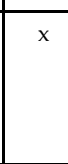 & & I & & $\mathrm{Fc}$ & & A & $\mathrm{R}$ & & $\mathrm{R}$ & $\mathrm{P}$ & c & \begin{tabular}{|l|l|}
$\begin{array}{c}\text { A-Program } \\
\text { Mgr }\end{array}$ \\
\end{tabular} \\
\hline \begin{tabular}{l|}
$\begin{array}{l}\text { System Operations } \\
\text { and Maintenance } \\
\text { Plan }\end{array}$ \\
\end{tabular} & 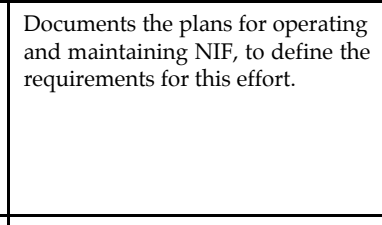 & 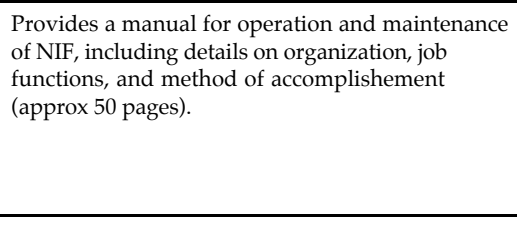 & 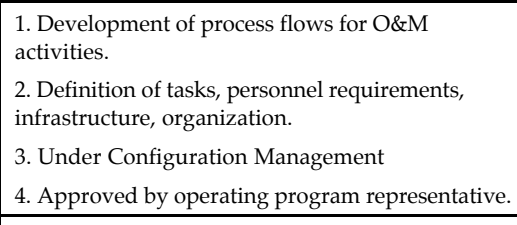 & 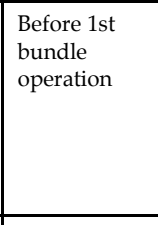 & $x$ & $x$ & $x^{x}$ & $x$ & $x$ & $x$ & $\times$ & $\mathrm{x}$ & $\times$ & $\times$ & $x^{x}$ & & & $\mathrm{~F}$ & & c & ${ }_{\mathrm{R}}$ & ${ }_{R}^{R}$ & \begin{tabular}{|l|l|l|} 
\\
\end{tabular} & 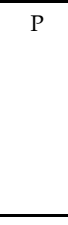 & & 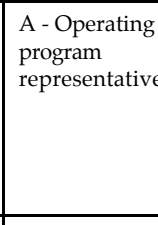 \\
\hline osps & 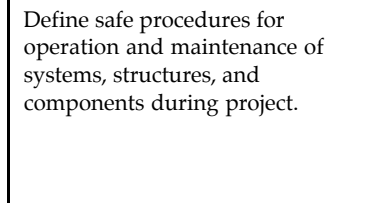 & $\begin{array}{l}\text { Standard LLNL format and content. } \\
\end{array}$ & 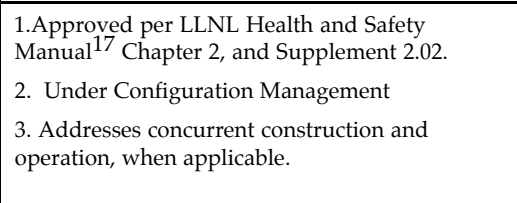 & $\begin{array}{l}\text { Before } \\
\text { subsystem } \\
\text { operation }\end{array}$ & & & $\begin{array}{l}.1 \\
.2 \\
.3 \\
.4 \\
.5\end{array}$ & .1 & ${ }^{4}$ & ${ }_{9}^{8}$ & .2 & .2 & .2 & & & \begin{tabular}{|l}
$P$ \\
\end{tabular} & $\mathrm{R}$ & A & & & $\mathrm{R}$ & & & $\mathrm{P}$ & & \begin{tabular}{|l} 
G.LLNL Hece \\
Manual
\end{tabular} \\
\hline
\end{tabular}




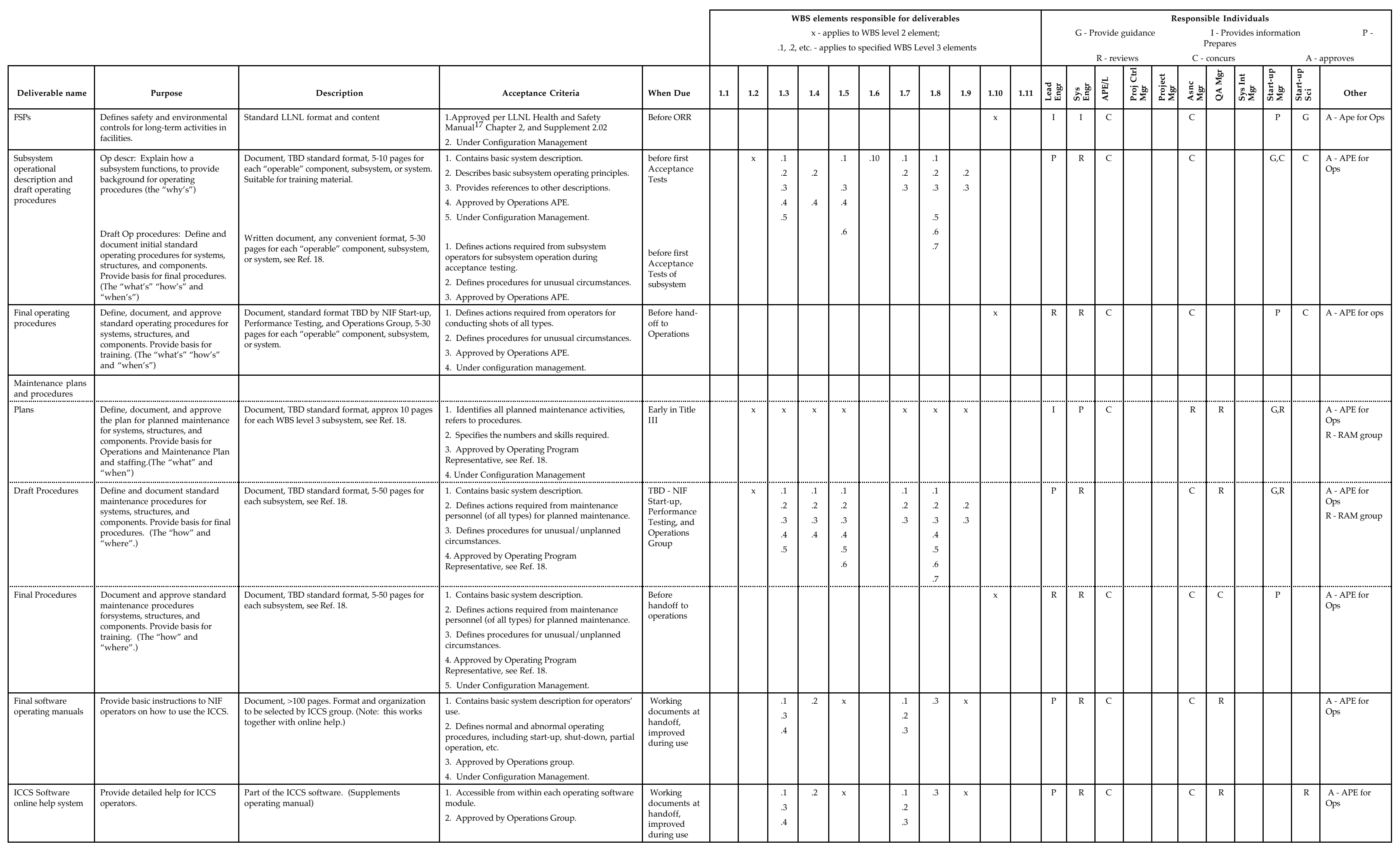




\begin{tabular}{|c|c|c|c|c|c|c|c|c|c|c|c|c|c|c|c|c|c|c|c|c|c|c|c|c|c|c|}
\hline \multirow[b]{2}{*}{ Deliverable name } & \multirow[b]{2}{*}{ Purpose } & \multirow[b]{2}{*}{ Description } & \multirow[b]{2}{*}{ Acceptance Criteria } & \multirow[b]{2}{*}{ When Due } & \multicolumn{11}{|c|}{$\begin{array}{c}\text { WBS elements responsible for deliverables } \\
\text { x-applies to WBS level } 2 \text { element; } \\
\text {.1, 2, etc. - applies to specified WBS Level } 3 \text { elements }\end{array}$} & \multicolumn{4}{|c|}{$\begin{array}{c}\text { G- Provide guidance } \\
\text { R-reviews } \\
\end{array}$} & \multicolumn{6}{|c|}{$\begin{array}{ccc}\text { I- Provides information } & \text { P- } \\
\text { Prepares } & \text { P-approves } \\
\text { C- concurs } & \text { A- apres }\end{array}$} & A-approves \\
\hline & & & & & 1.1 & 1.2 & 1.3 & 1.4 & 1.5 & 1.6 & 1.7 & 1.8 & 1.9 & 1.10 & 1.11 & 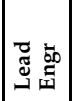 & 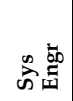 & 黄 & 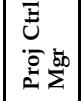 & 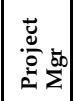 & 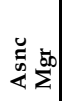 & $\begin{array}{l}\text { 然 } \\
\text { and } \\
d\end{array}$ & 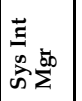 & 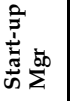 & $\mid$ & Other \\
\hline $\begin{array}{l}\begin{array}{l}\text { Training } \\
\text { Development }\end{array} \\
\end{array}$ & $\begin{array}{l}\text { Participate as subject-matter } \\
\text { experts in the analysis, design, } \\
\text { development, and implementation } \\
\text { of performance-based training. }\end{array}$ & $\begin{array}{l}\text { Attend selected operations group meetings, } \\
\text { prepare and deliver } 1 \text { training class for each } \\
\text { operable or maintainable unit. }\end{array}$ & $\begin{array}{l}\text { 1. Approved by Operating Program } \\
\text { Representative. }\end{array}$ & 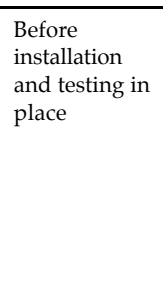 & & $x$ & $\begin{array}{l}.1 \\
.2 \\
.3 \\
.4 \\
.5\end{array}$ & \begin{tabular}{l|}
.1 \\
2 \\
3 \\
.4
\end{tabular} & \begin{tabular}{l|}
.1 \\
.3 \\
.4 \\
.5 \\
.6
\end{tabular} & $\begin{array}{l}.8 \\
.9 \\
.10\end{array}$ & $\begin{array}{l}.1 \\
.2 \\
3\end{array}$ & $\begin{array}{l}.1 \\
.2 \\
.3 \\
.4 \\
.5 \\
.6 \\
.7\end{array}$ & $\begin{array}{l}.1 \\
.2 \\
.3\end{array}$ & $x$ & & \begin{tabular}{|l|}
$\mathrm{I}, \mathrm{P}$ \\
\end{tabular} & \begin{tabular}{|l|}
$I, R$ \\
\end{tabular} & C & & & & & & $P$ & $\mathrm{R}$ & \begin{tabular}{|l} 
A-Ape for Ops \\
\end{tabular} \\
\hline $\begin{array}{l}\text { completed mgmt } \\
\text { statr-up reviess for } \\
\text { each bundle }\end{array}$ & $\begin{array}{l}\text { Confirm readiness to handoff each } \\
\text { bundle to Operating Program. }\end{array}$ & $\begin{array}{l}\text { Internal review meeting with identified review } \\
\text { commitite Standard checklist revieved, action } \\
\text { items assigned, tracked and closed out. }\end{array}$ & 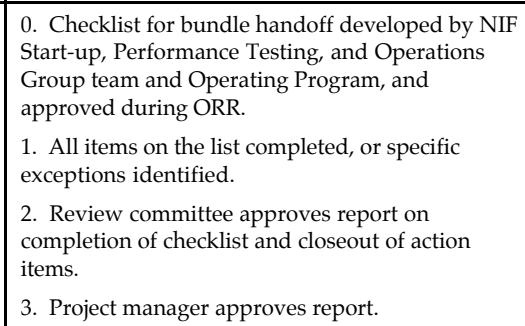 & $\begin{array}{l}\text { before } \\
\text { handoff of } \\
\text { each bundle } \\
\text { to herating } \\
\text { Program }\end{array}$ & & & & & & & & & & $x$ & & \begin{tabular}{|l|}
$\mathrm{I}$ \\
\end{tabular} & $\begin{array}{ll}\mathrm{I} \\
\end{array}$ & $\begin{array}{l}\mathrm{R} \\
\mathrm{R}\end{array}$ & & $\mathrm{A}$ & C & $\mathrm{R}$ & $\mathrm{R}$ & $P$ & c & $\begin{array}{l}\begin{array}{l}\text { C-Review } \\
\text { committee }\end{array} \\
\end{array}$ \\
\hline Configuration data & & & & & & & & & & & & & & & & & & & & & & & & & & \\
\hline 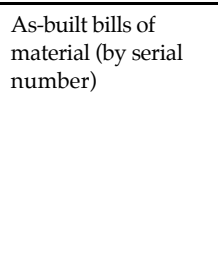 & $\begin{array}{l}\text { Define the actual configuration of } \\
\text { all III hardware, by serial number } \\
\text { where appropriate. }\end{array}$ & $\begin{array}{l}\text { Standard report produced by NIF Configuration } \\
\text { Management Information System on request. } \\
\text { Indentured parts list format or equal. } \\
\text { See Appendix B-1. }\end{array}$ & \begin{tabular}{|l} 
1. Produced by NIF Configuration Management \\
Information Systemu. \\
2. Under Configuration Managagement. \\
3. Audited/verified by QA (sampling TBD).
\end{tabular} & $\begin{array}{l}\text { maintained } \\
\text { throughout } \\
\text { Title IIl due } \\
\text { at end of } \\
\text { acceptance } \\
\text { test fonceach } \\
\text { bundle }\end{array}$ & & $\mathrm{x}$ & $\begin{array}{l}.1 \\
.2 \\
.3 \\
.4 \\
.5\end{array}$ & \begin{tabular}{l|}
.1 \\
.2 \\
.3 \\
.4
\end{tabular} & \begin{tabular}{l|}
.1 \\
.3 \\
.4 \\
.5 \\
.6
\end{tabular} & & $\begin{array}{l}.1 \\
.2 \\
3\end{array}$ & \begin{tabular}{l|}
.1 \\
.2 \\
.3 \\
.4 \\
.5 \\
.6 \\
.7
\end{tabular} & $\begin{array}{l}.1 \\
.2 \\
.3\end{array}$ & & & \begin{tabular}{|l|}
$\mathrm{I}, \mathrm{P}$ \\
\end{tabular} & \begin{tabular}{ll|}
$I, R$ \\
\end{tabular} & & & & & \begin{tabular}{ll|}
$\mathrm{R}, \mathrm{A}$ \\
\end{tabular} & $\mathrm{L}, \mathrm{R}$ & & & $\begin{array}{l}\begin{array}{l}P-C M \text { staff } \\
\text { (on demand) }\end{array} \\
\end{array}$ \\
\hline 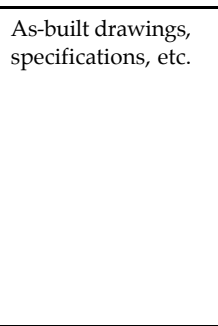 & $\begin{array}{l}\text { Define the actual configuration of } \\
\text { all NIF hardware. }\end{array}$ & $\begin{array}{l}\text { Standard report produced by NIF Configuration } \\
\text { Management Information System on request. } \\
\text { Indentured parts list with "described by" } \\
\text { relationships, or qual. } \\
\text { See Appendix B-2.2. }\end{array}$ & \begin{tabular}{|l} 
1. Produced by NIF Configuration Management \\
Information System. \\
2. Under Configuration Management. \\
3. Audited / verified by QA (sampling TBD). \\
\end{tabular} & $\begin{array}{l}\text { maintained } \\
\text { throughout } \\
\text { Tritle IIt, une } \\
\text { at end of } \\
\text { acceptance } \\
\text { test ton each } \\
\text { bundle, } \\
\text { revised as } \\
\text { requd during } \\
\text { operational } \\
\text { testing }\end{array}$ & & $x$ & $\begin{array}{l}.1 \\
.2 \\
.3 \\
.4 \\
.5\end{array}$ & \begin{tabular}{l|}
.1 \\
.2 \\
.3 \\
.4
\end{tabular} & \begin{tabular}{l|}
.1 \\
.2 \\
.3 \\
.4 \\
.5 \\
.6
\end{tabular} & .10 & $\begin{array}{l}1 \\
.2 \\
3\end{array}$ & $\begin{array}{l}.1 \\
.2 \\
.3 \\
.4 \\
.5 \\
.6 \\
.7\end{array}$ & $\begin{array}{l}.1 \\
.2 \\
.3\end{array}$ & & & \begin{tabular}{|l|}
$\mathrm{P}, \mathrm{A}$ \\
\end{tabular} & \begin{tabular}{c|c|}
$P, R$, \\
$A$
\end{tabular} & $\begin{array}{c}\mathrm{R}, \mathrm{C}, \mathrm{A} \\
\mathrm{A}\end{array}$ & & & & $R, A$ & \begin{tabular}{|l|}
$\mathrm{R}$ \\
\end{tabular} & & & \begin{tabular}{|l} 
P-CM staff \\
(on demand) \\
A- CCBA (high \\
level items \\
only)
\end{tabular} \\
\hline $\begin{array}{l}\text { Software Release } \\
\text { Descriptions }\end{array}$ & $\begin{array}{l}\text { Document sufficient information } \\
\text { to allow maintenance, } \\
\text { modification, upgrade and trouble- } \\
\text { shooting of ICCS software. }\end{array}$ & $\begin{array}{l}\text { Document, 100-1000 pages. } \\
\text { Thisis the product of the Physical Configuration } \\
\text { Audit (PCA) defined by the Software CM Plan19 }\end{array}$ & $\begin{array}{l}\text { 1. Defines sufficient information to allow } \\
\text { maintenance, modification, upgrade and trouble- } \\
\text { shooting of IICCS Softtware. } \\
\text { 2. Approved by Operations group. }\end{array}$ & \begin{tabular}{|l|}
$\begin{array}{l}\text { At end of } \\
\text { acceptance } \\
\text { tests, when } \\
\text { modifications } \\
\text { are complete }\end{array}$ \\
\end{tabular} & & & $\begin{array}{l}.1 \\
.3 \\
.4\end{array}$ & .2 & \begin{tabular}{l|}
.2 \\
.3 \\
.4 \\
.5 \\
.6
\end{tabular} & & $\begin{array}{l}1 \\
.2 \\
.3\end{array}$ & .3 & & & & \begin{tabular}{|l|}
$P$ \\
\end{tabular} & $\begin{array}{ll}R \\
\end{array}$ & $\mathrm{C}$ & & & $\mathrm{C}$ & $\mathrm{R}$ & & & & \\
\hline $\begin{array}{l}\text { Configuration audit } \\
\text { reports }\end{array}$ & $\begin{array}{l}\text { Demonstrate correspondence } \\
\text { betereen the actual configuration } \\
\text { and as-built documentations } \\
\end{array}$ & 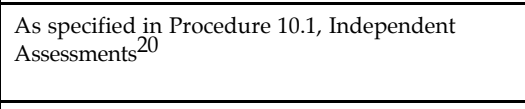 & \begin{tabular}{|l|}
$\begin{array}{l}\text { Verifies compliance with Titel II change control } \\
\text { process and NIF Configuration Management } \\
\text { Plan? }\end{array}$ \\
\end{tabular} & as req'd & $x$ & & & & & & & & & & & \begin{tabular}{|l|}
$\mathrm{I}$ \\
\end{tabular} & $\mathrm{I}$ & $\begin{array}{l}\mathrm{I} \\
\end{array}$ & & & $\begin{array}{ll}\mathrm{G}, \mathrm{A} \\
\end{array}$ & $P$ & $\begin{array}{lll}R, I \\
\end{array}$ & & & \\
\hline 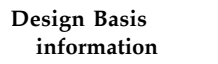 & & & & & & & & & & & & & & & & & & & & & & & & & & \\
\hline project files & $\begin{array}{l}\text { Maintain complete information on } \\
\text { the NII project and the resulting } \\
\text { hardware, for future use by } \\
\text { operations for troubleshooting, } \\
\text { maintenance, upgrading, etc. }\end{array}$ & 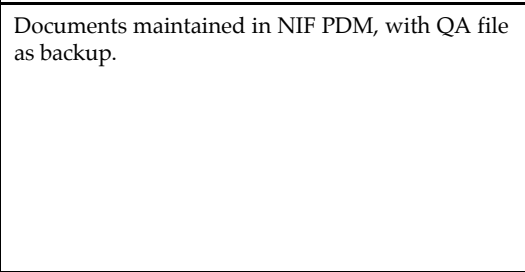 & 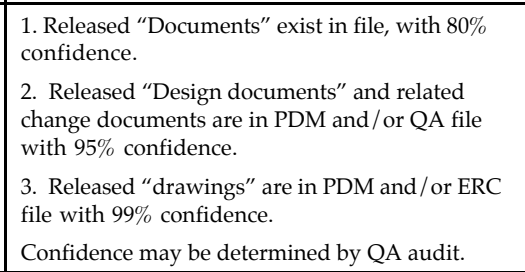 & \begin{tabular}{|l|} 
continuous \\
\end{tabular} & (all) & (all) & $\begin{array}{l}\text { (all) } \\
\text { (a) }\end{array}$ & (all) & (all) & (all) & $($ (all) & (all) & (all) & (all) & (all) & $\begin{array}{c}, R, R \\
{ }_{A} \\
\end{array}$ & $\begin{array}{c}\mathrm{P}, \mathrm{R}, \\
\mathrm{A}\end{array}$ & $\begin{array}{c}P, R, \\
A\end{array}$ & \begin{tabular}{|c|c|}
$P, R$, \\
$A$
\end{tabular} & $\begin{array}{c}P, R_{,} \\
A\end{array}$ & $\begin{array}{l}, R_{,}, \\
A A\end{array}$ & $\begin{array}{c}, R_{,} \\
A_{A}\end{array}$ & $\begin{array}{c}\mathrm{P}, \mathrm{R}, \\
\mathrm{A}\end{array}$ & \begin{tabular}{c|c}
$P, R$, \\
$A$
\end{tabular} & $\begin{array}{c}\mathrm{P}, \mathrm{R}, \\
\mathrm{A}\end{array}$ & \begin{tabular}{|l|} 
P,R,A-all \\
project \\
personnel \\
A-QA audits
\end{tabular} \\
\hline
\end{tabular}




\begin{tabular}{|c|c|c|c|c|c|c|c|c|c|c|c|c|c|c|c|c|c|c|c|c|c|c|c|c|c|c|}
\hline & & & & & \multicolumn{11}{|c|}{$\begin{array}{l}\text { WBS elements responsible for deliverables } \\
\text { x-applies to WBB level e element; } \\
\text {.1, 2, etc. - applies to specified WBS Level } 3 \text { elements }\end{array}$} & & & $\begin{array}{c}\text { rovide } \\
\text { R- rev }\end{array}$ & guidance & & & $\begin{array}{l}\text { Ile Indi } \\
\text { I- - Prov } \\
\text { repares } \\
\text { oncurs }\end{array}$ & $\begin{array}{l}\text { Hividua } \\
\text { vides in } \\
;\end{array}$ & fis & & approves \\
\hline Deliverable name & Purpose & Description & Acceptance Criteria & When Due & 1.1 & 1.2 & 1.3 & 1.4 & 1.5 & 1.6 & 1.7 & 1.8 & 1.9 & 1.10 & 1.11 & 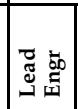 & $\begin{array}{ccc}0 \\
n \\
n\end{array}$ & 黄 & 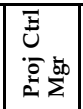 & 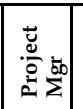 & 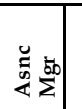 & 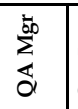 & 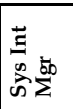 & 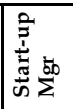 & 童 & Other \\
\hline CAD models & $\begin{array}{l}\text { Maintain complete information on } \\
\text { NIF hardware, for future use by } \\
\text { operations for troubleshooting, } \\
\text { maintenance, upgrading, etc. }\end{array}$ & $\begin{array}{l}\text { ProE and AutoCAD 3D models maintained in } \\
\text { PropDM (or equal). } \\
\text { CF models in Intergraph. }\end{array}$ & $\begin{array}{l}\text { 1. Modeles “released” in ProPDM (CF in } \\
\text { Integraph). } \\
\text { 2. Ooperaions design supervisor approves receipt } \\
\text { of models. }\end{array}$ & \begin{tabular}{|l}
$\begin{array}{l}\text { by end of } \\
\text { project }\end{array}$ \\
\end{tabular} & & (all) & (all) & (all) & & (all) & (all) & (all) & (all) & & & & & & & & & & & & & $\begin{array}{l}\text { P,R-designers } \\
\text { A-Ops design } \\
\text { supervisor }\end{array}$ \\
\hline $\begin{array}{l}\text { Physics basis } \\
\text { document, } \\
\text { updated with } \\
\text { actual test results }\end{array}$ & \begin{tabular}{|l|} 
Provide physics basis necessary to \\
operate laser within safe limits. \\
Used for veluatuating proposed shot \\
parameters.
\end{tabular} & $\begin{array}{l}\text { Document, approx } 100 \text { pages. Format and } \\
\text { organization TBD by System Integration. } \\
\text { Includes test results for Bu31, and as much of C1 } \\
\text { and C2 as possible. }\end{array}$ & 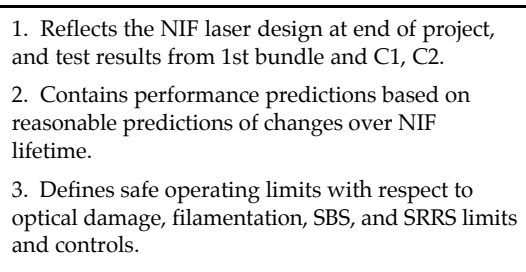 & 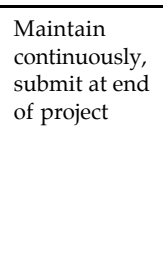 & $\mathrm{x}$ & & & & & & & & & & & & & & & & & & & & A & $\begin{array}{l}\text { P,R-Analyst } \\
\text { team } \\
\text { A- Project } \\
\text { Scientist }\end{array}$ \\
\hline $\begin{array}{l}\text { Baseline Prop92 } \\
\text { moded (or } \\
\text { equivalent) }\end{array}$ & 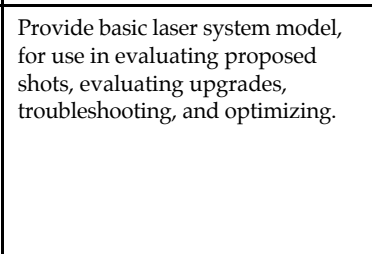 & $\begin{array}{l}\text { Computer input files, combined with controlled } \\
\text { version of codes. }\end{array}$ & $\begin{array}{l}\text { 1. Input file reflects as-built configuration with } \\
\text { reasonable filedity. } \\
\text { 2. Input file is under } \mathrm{CM} \text {. } \\
\text { 3. Analysis code is under } \mathrm{CM} \text {. } \\
\text { 4. NIF Project Scientist approves. } \\
\text { 5. Analysts supporting operations acknowledge } \\
\text { receipto om model. }\end{array}$ & 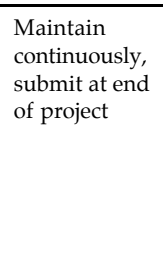 & $\mathrm{x}$ & & & & & & & & & & & & & & & & & & & & A & $\begin{array}{l}\text { P,R-Analyst } \\
\text { team } \\
\text { A- Project } \\
\text { Scientist }\end{array}$ \\
\hline $\begin{array}{l}\text { Title III } \\
\text { Engineering Basis } \\
\text { Book (includes } \\
\text { other deliverables) }\end{array}$ & 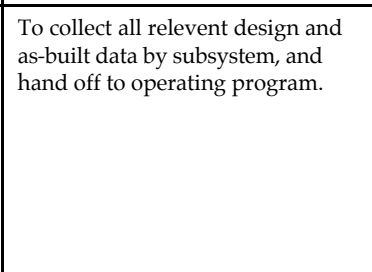 & 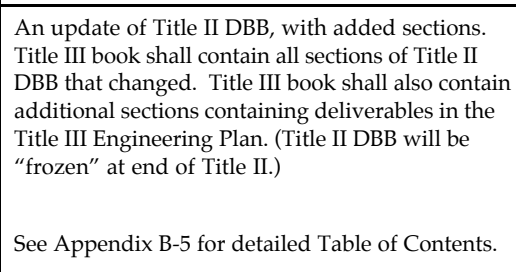 & $\begin{array}{l}\text { 1. Review by QA. } \\
\text { 2. Approved by APE. } \\
\text { 3. Accepted by operating program. }\end{array}$ & $\begin{array}{l}\text { At end of } \\
\text { project }\end{array}$ & & & $\begin{array}{l}.1 \\
.2 \\
.3 \\
.4 \\
.5\end{array}$ & $\begin{array}{l}.1 \\
.2 \\
.3 \\
.4\end{array}$ & $\begin{array}{l}.1 \\
.2 \\
.3 \\
.4 \\
.5 \\
.6\end{array}$ & & $\begin{array}{l}.1 \\
.2 \\
.3\end{array}$ & $\begin{array}{l}1.1 \\
.2 \\
.3 \\
.4 \\
.5 \\
.6 \\
.7\end{array}$ & $\begin{array}{l}.1 \\
.2 \\
.3\end{array}$ & & & 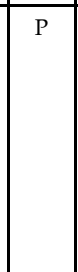 & $\mathrm{R}$ & $\mathrm{A}$ & & & $\mathrm{R}$ & $\mathrm{R}$ & $\mathrm{R}$ & $\mathrm{R}$ & $\bar{R}$ & \\
\hline $\begin{array}{l}\text { Fracture-Control } \\
\text { Plans for britle } \\
\text { vacuum barriers }\end{array}$ & 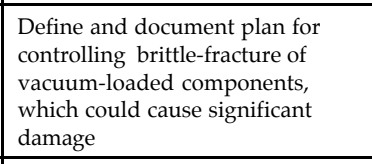 & Per new LLNL Mechanical Engineering guidlines & $\begin{array}{l}\text { see LLNL ME Department guidlines } \\
\text { (and under Configuration Management) }\end{array}$ & & & & & & & & & & & & & & & & & & & & & & & \\
\hline $\begin{array}{l}\text { Supporting } \\
\text { calculations }\end{array}$ & 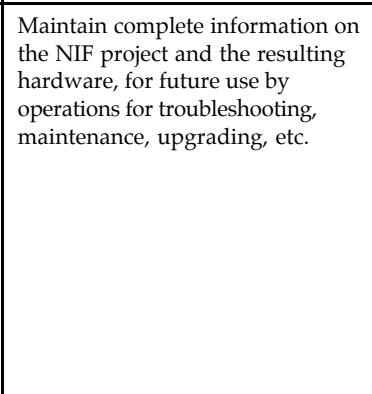 & \begin{tabular}{|l} 
Memos, safety notes, reports, and other \\
documents as appropriate,
\end{tabular} & $\begin{array}{l}\text { 1. In NIF PDM. } \\
\text { 2. Checked and approved. }\end{array}$ & Continuous & $\mathrm{s}$ & $\mathrm{x}$ & $\begin{array}{l}.1 \\
.2 \\
.3 \\
.4 \\
.5\end{array}$ & $\begin{array}{l}.1 \\
.2 \\
3 \\
.4\end{array}$ & $\begin{array}{l}.1 \\
.2 \\
.3 \\
.4 \\
.5 \\
.6\end{array}$ & $\begin{array}{l}1 \\
.2 \\
.3 \\
.4 \\
.5 \\
.6 \\
.7 \\
.8 \\
.9 \\
.10 \\
\end{array}$ & $\begin{array}{l}.1 \\
.2 \\
.3\end{array}$ & $\begin{array}{l}.1 \\
.2 \\
.3 \\
.4 \\
.5 \\
.6 \\
.7\end{array}$ & $\begin{array}{l}.1 \\
.2 \\
.3\end{array}$ & & & $\begin{array}{c}\mathrm{P}, \mathrm{R} \\
\mathrm{A}\end{array}$ & $\begin{array}{c}\mathrm{P}, \mathrm{R}, \\
\mathrm{A}\end{array}$ & $\begin{array}{c}P, R, \\
A\end{array}$ & & & & & & & & $\begin{array}{l}\text { others per } \\
\text { LLNL policy }\end{array}$ \\
\hline
\end{tabular}




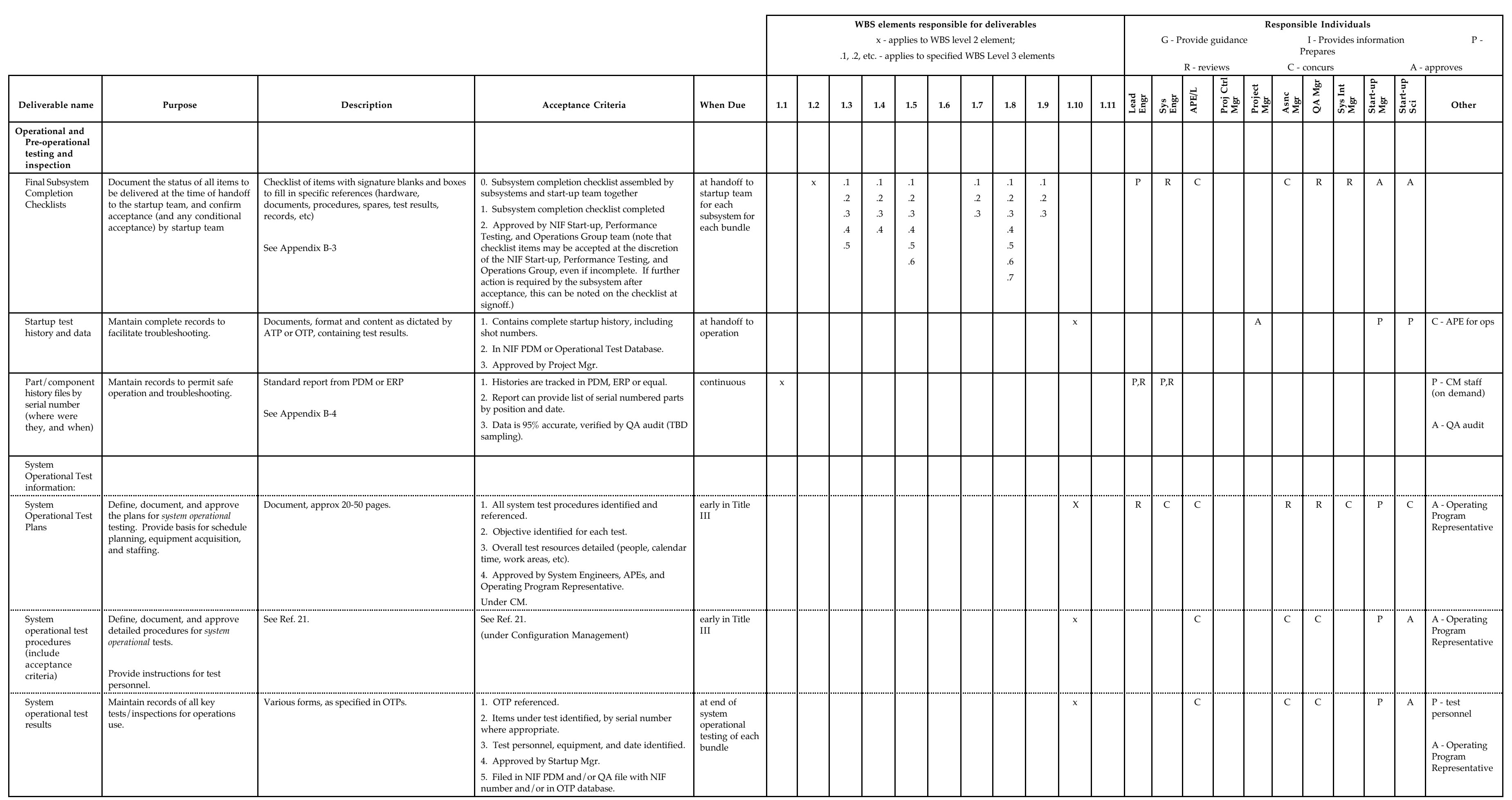




\begin{tabular}{|c|c|c|c|c|c|c|c|c|c|c|c|c|c|c|c|c|c|c|c|c|c|c|c|c|c|}
\hline \multirow[b]{2}{*}{ Deliverable name } & \multirow[b]{2}{*}{ Purpose } & \multirow[b]{2}{*}{ Description } & \multirow[b]{2}{*}{ Acceptance Criteria } & \multirow[b]{2}{*}{ When Due } & \multicolumn{11}{|c|}{ 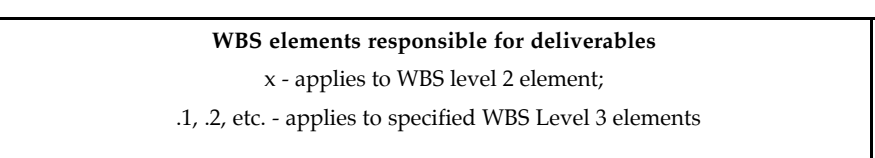 } & \multicolumn{10}{|c|}{ 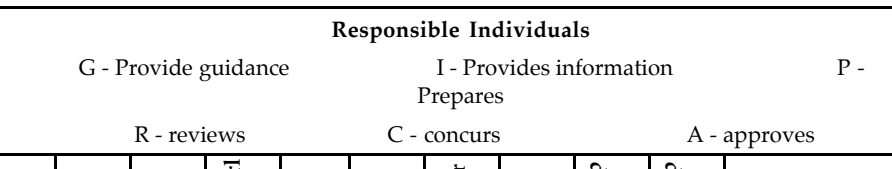 } \\
\hline & & & & & 1.1 & 1.2 & 1.3 & 1.4 & 1.5 & 1.6 & 1.7 & 1.8 & 1.9 & 1.10 & 1.11 & 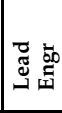 & 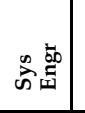 & 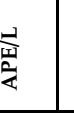 & 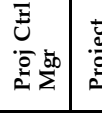 & $\begin{array}{ll}5 \\
\frac{5}{2} \\
2\end{array}$ & \begin{tabular}{|l}
$\frac{5}{5}$ \\
$\frac{5}{2}$ \\
\end{tabular} & 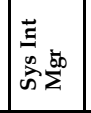 & $\begin{array}{lllll}0 & 1\end{array}$ & 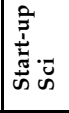 & other \\
\hline \multicolumn{26}{|l|}{ 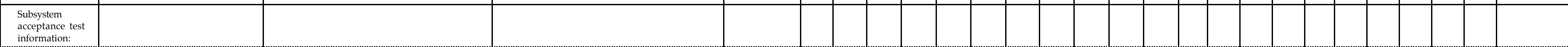 } \\
\hline $\begin{array}{l}\text { Subbsysem } \\
\text { accertance test } \\
\text { plans }\end{array}$ & 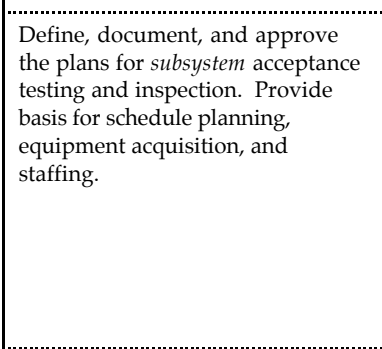 & Document, approx 5-20 pages for each subsystem. & 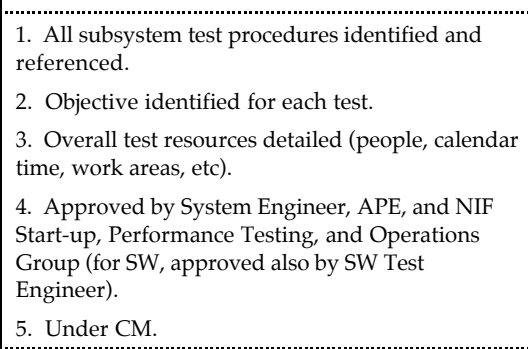 & 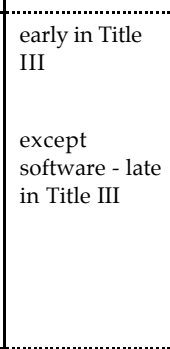 & & x & $\begin{array}{l}.1 \\
.2 \\
3 \\
3 \\
.5 \\
.5\end{array}$ & $\begin{array}{l}1 \\
2 \\
3 \\
.4\end{array}$ & $\begin{array}{l}.1 \\
2 \\
3 \\
.4 \\
.5 \\
.6\end{array}$ & $\begin{array}{l}1 \\
2 \\
.3 \\
4 \\
.5 \\
.6 \\
7\end{array}$ & $\begin{array}{l}1 \\
2 \\
2 \\
3\end{array}$ & $\begin{array}{l}.1 \\
.2 \\
.3 \\
4 \\
.5 \\
.6 \\
7\end{array}$ & $\begin{array}{l}3 \\
2 \\
3 \\
3\end{array}$ & & & P & c & c & & $\mathrm{R}$ & R & c & $\mathrm{G}, \mathrm{A}$ & $\mathrm{G}, \mathrm{C}$ & 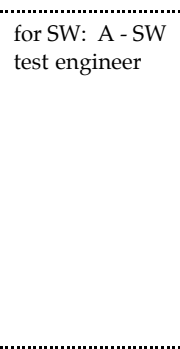 \\
\hline 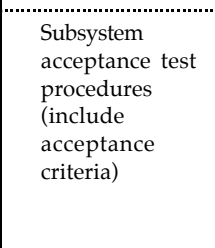 & 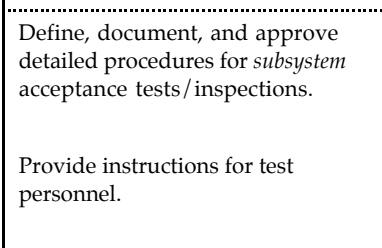 & See Ref. 21. & $\begin{array}{l}\text { See Ret 21. } \\
\text { (under Configuration Management) }\end{array}$ & 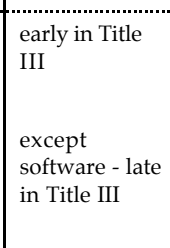 & & $x^{x}$ & $\begin{array}{l}1 \\
2 \\
.3 \\
3 \\
4 \\
5\end{array}$ & $\begin{array}{l}1 \\
.2 \\
.3 \\
.4\end{array}$ & $\begin{array}{l}.1 \\
.2 \\
3 \\
4 \\
.5 \\
.6\end{array}$ & $\begin{array}{l}1 \\
2 \\
2 \\
3 \\
4 \\
5\end{array}$ & $\begin{array}{l}1 \\
.2 \\
.3\end{array}$ & $\begin{array}{l}.1 \\
.2 \\
3 \\
4 \\
5\end{array}$ & $\begin{array}{l}3 \\
1 \\
2 \\
3\end{array}$ & & & P & A & & & $\mathrm{G}, \mathrm{C}$ & c & & $\mathrm{G,C}$ & G & $\begin{array}{l}\text { for SW: AN: A, } \\
\text { SW tets } \\
\text { enginer }\end{array}$ \\
\hline $\begin{array}{l}\text { Subsystem } \\
\text { acceptance test } \\
\text { results }\end{array}$ & 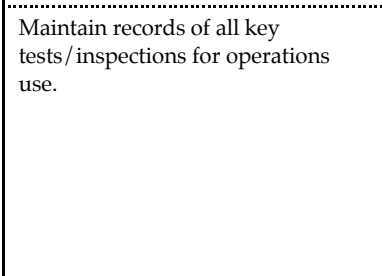 & Various forms, as specified in ATPs. & 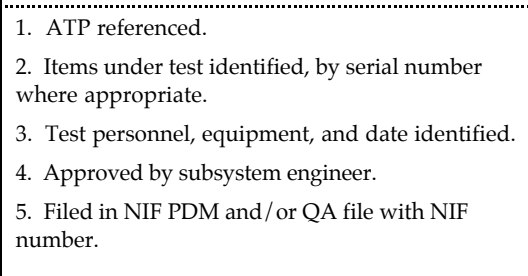 & $\begin{array}{l}\text { at end of } \\
\text { subsystem } \\
\text { acceptance } \\
\text { tetsing of each } \\
\text { bundle }\end{array}$ & & x & $\begin{array}{l}1 \\
.1 \\
.3 \\
3 \\
4 \\
5\end{array}$ & $\begin{array}{l}.1 \\
.2 \\
.3 \\
.4\end{array}$ & $\begin{array}{l}1 \\
.2 \\
.3 \\
.4 \\
.5 \\
.6\end{array}$ & $\begin{array}{l}1 \\
.2 \\
3 \\
4 \\
5\end{array}$ & $\begin{array}{l}1 \\
.2 \\
.3\end{array}$ & $\begin{array}{l}.1 \\
.2 \\
3 \\
4\end{array}$ & $\begin{array}{l}1 \\
2 \\
3 \\
3\end{array}$ & & & $P, R$ & R & R & & & & & $\mathrm{A}^{\mathrm{A}}$ & R & $\begin{array}{l}\text { P-test } \\
\text { personnel } \\
\text { for SW: P-SW } \\
\text { test tengineer }\end{array}$ \\
\hline \multicolumn{26}{|l|}{ 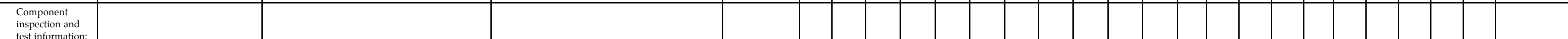 } \\
\hline plans & 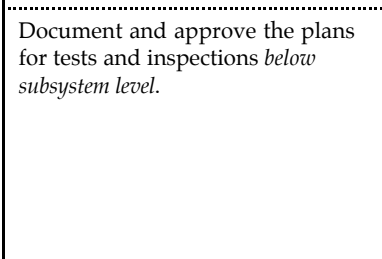 & $\begin{array}{l}\text { IIf updated atere Titite II) Document, } 5 \text { pages for } \\
\text { each subsystem. }\end{array}$ & 1. & 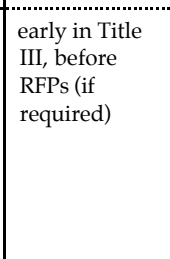 & & $x^{x}$ & $\begin{array}{l}1 \\
.1 \\
.3 \\
3 \\
.5 \\
.5\end{array}$ & $\begin{array}{l}.1 \\
.2 \\
.3 \\
.4\end{array}$ & $\begin{array}{l}.1 \\
.2 \\
.3 \\
4 \\
.5 \\
.6\end{array}$ & $\begin{array}{l}1 \\
2 \\
2 \\
3 \\
4 \\
5\end{array}$ & $\begin{array}{l}1 \\
2 \\
3 \\
3\end{array}$ & $\begin{array}{l}1 \\
2 \\
3\end{array}$ & $\begin{array}{l}1 \\
2 \\
3\end{array}$ & & & P & A & & & & G & $\mathrm{R}$ & & & \\
\hline procedures & 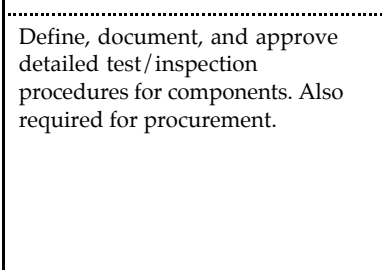 & $\begin{array}{l}\text { (If p pdated alter Titite III Document, } 1.5 \text { pages for for } \\
\text { each type of inspection. }\end{array}$ & 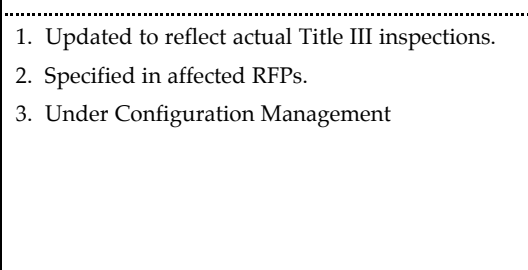 & 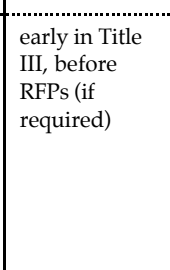 & & & $\begin{array}{l}1 \\
.2 \\
3 \\
4 \\
5 \\
5\end{array}$ & $\begin{array}{l}1 \\
2 \\
3 \\
4\end{array}$ & $\begin{array}{l}.1 \\
.2 \\
.3 \\
.4 \\
.5 \\
.6\end{array}$ & $\begin{array}{l}1 \\
2 \\
3 \\
4 \\
5\end{array}$ & $\begin{array}{l}1 \\
.2 \\
3\end{array}$ & 3 & $\begin{array}{l}3.1 \\
: 2 \\
3\end{array}$ & & & P & A & & & & G & $\mathrm{R}$ & & & \\
\hline results & $\begin{array}{l}\text { Maintain records of all key data for } \\
\text { operations use. }\end{array}$ & Various forms as convenient, include raw data. & 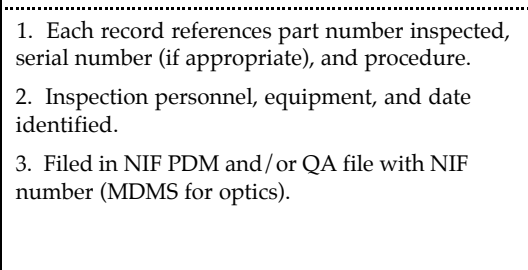 & $\begin{array}{l}\text { at end of } \\
\text { subsystem } \\
\text { acceptance } \\
\text { testing for } \\
\text { each bundle }\end{array}$ & $x^{x}$ & & $\begin{array}{l}1 \\
.1 \\
.3 \\
3 \\
4 \\
.5\end{array}$ & .3 & $\begin{array}{l}1 \\
.2 \\
.3 \\
.4 \\
.5 \\
.6\end{array}$ & $\begin{array}{l}1 \\
.2 \\
3 \\
.4 \\
5\end{array}$ & $\begin{array}{l}1 \\
2 \\
3\end{array}$ & .3 & $\begin{array}{l}1 \\
2 \\
3\end{array}$ & & & P & A & & & & G & $\mathrm{R}$ & & & \\
\hline
\end{tabular}




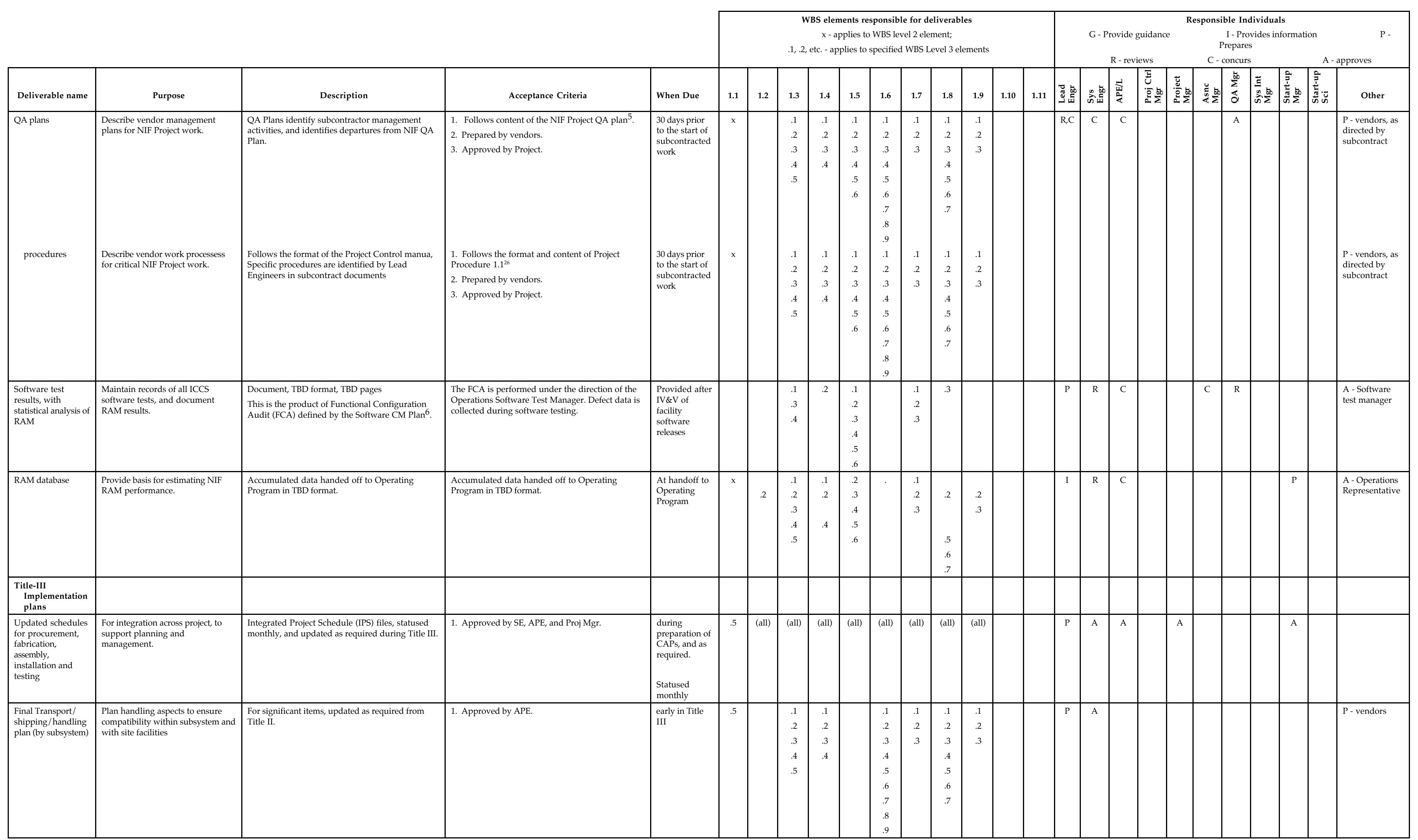




\begin{tabular}{|c|c|c|c|c|c|c|c|c|c|c|c|c|c|c|c|c|c|c|c|c|c|c|c|c|c|}
\hline & & & & & \multicolumn{11}{|c|}{$\begin{array}{l}\text { WBS elements responsible for deliverables } \\
x \text { x applies to WSB level } 2 \text { element; } \\
\text {.1, 2, etc. - applies to specified WBS Level } 3 \text { elements }\end{array}$} & & $\begin{array}{r}\text { G-Pro } \\
R\end{array}$ & $\begin{array}{l}\text { ovide gu } \\
\text { R- revier }\end{array}$ & $\begin{array}{l}\text { uidance } \\
\text { ews }\end{array}$ & $\operatorname{Res}_{1}$ & $\begin{aligned} \text { ponsible } \\
\text { I- } \\
\text { Prep } \\
\text { C- conc }\end{aligned}$ & $\begin{array}{l}\text { Individ } \\
\text { Provides } \\
\text { pares } \\
\text { currs }\end{array}$ & $\begin{array}{l}\text { iduals } \\
\text { les inform } \\
\end{array}$ & mation & pproves \\
\hline Deliverable name & Purpose & Description & Acceptance Criteria & When Due & 1.1 & 1.2 & 1.3 & 1.4 & 1.5 & 1.6 & 1.7 & 1.8 & 1.9 & 1.10 & 1.11 & 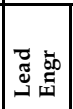 & 品管 & 善 & 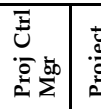 & 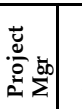 & \begin{tabular}{l|l} 
\\
\end{tabular} & & 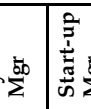 & 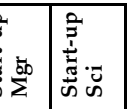 & Other \\
\hline $\begin{array}{l}\text { Final Assembly/ } / \\
\text { intslathion/ } \\
\text { acceptance plan }\end{array}$ & $\begin{array}{l}\text { For integration, planning, and } \\
\text { management of project. }\end{array}$ & Updated as required from Title II. & 1. Approved by APE. & $\begin{array}{l}\text { early in Title } \\
\text { III }\end{array}$ & 5.5 & & $\begin{array}{l}.1 \\
.2 \\
.3 \\
.4 \\
.5\end{array}$ & $\begin{array}{l}.1 \\
.2 \\
.3 \\
.4\end{array}$ & & & $\begin{array}{l}.1 \\
.2 \\
.3\end{array}$ & \begin{tabular}{l|}
.1 \\
.2 \\
.3 \\
.4 \\
.5 \\
.6 \\
.7
\end{tabular} & $\begin{array}{l}.1 \\
.2 \\
.3\end{array}$ & & & \begin{tabular}{|l|}
$P$ \\
\end{tabular} & $\mathrm{R}$ & $\mathrm{C}$ & & & & & \begin{tabular}{l|l}
$\mathrm{R}$ & $\mathrm{C}$ \\
\end{tabular} & c & A-APE (ops) \\
\hline $\begin{array}{c}\begin{array}{c}\text { Management } \\
\text { deliverables }\end{array} \\
\text { nat }\end{array}$ & & & & & & & & & & & & & & & & & & & & & & & & & \\
\hline CAPs (yearly) & Management of work packages. & $\begin{array}{l}\text { same as Title II. Workscope statement, CAP } \\
\text { summary sheet Primavera schedulu at } \\
\text { appropriate evel of detail for tracking, NPS input } \\
\text { sheet. }\end{array}$ & $\begin{array}{l}\text { 1. Approval by originator, }, \mathrm{SE}, \mathrm{APE} \text {, and project } \\
\text { control manager. }\end{array}$ & yearly & (all) & (all) & (all) & (all) & (all) & (all) & (all) & (all) & (all) & (all) & (all) & \begin{tabular}{|l|}
$P$ \\
\end{tabular} & $\mathrm{R}$ & $\mathrm{C}$ & A & A & & & & & \\
\hline $\begin{array}{l}\text { Monthly /quarterly } \\
\text { progress reports } \\
\text { (written and oral) } \\
\end{array}$ & Project reporting. & same as Titte II. & Approval of Senior Staff Engineer. & monthly & (all) & (all) & (all) & (all) & (all) & (all) & (all) & (all) & (all) & (all) & (all) & \begin{tabular}{|l|}
$P$ \\
\end{tabular} & $\mathrm{P}$ & $\mathrm{P}$ & $\mathrm{R}$ & A & $\mathrm{R}$ & $\mathrm{R}$ & P & P & \\
\hline
\end{tabular}




\subsection{Title III Schedule}

The NIF Project schedule baseline was approved at the end of Title I, as reflected in the Project Execution Plan ${ }^{23}$. This schedule baseline was founded on the detailed Integrated Project Schedule (IPS), which consists of interlinked schedule files. The IPS captures all schedule data for the Project, from long-range plans to detailed yearly work planning. Schedule activities are broken down at the level of detail appropriate for various applications. For yearly work planning and management, tasks are identified and milestones are selected for inclusion in Cost Account Plans. For installation planning and coordination, tasks are broken down into fine detail, to clarify interfaces and overlaps. The IPS is maintained by formal change-control procedures $^{24,25}$. For the most current version, consult the Project Lead Scheduler. 


\subsection{Title III Cost}

The NIF Project cost baseline was approved at the end of Title I, as reflected in the Project Execution Plan ${ }^{23}$. This cost baseline and the internal breakdown of the budget is maintained by the Project via formal changecontrol processes ${ }^{24}$. Detailed Cost Account Planning is performed approximately yearly, within this overall budget framework. For a listing of the Title III engineering budget, consult the Project Control Office. 


\subsection{References}

[1] NIF Title II Design Plan, NIF-1249.

[2] NIF Title I Design Plan, NIF-96-650

[3] NIF Site Management Plan, NIF-2564.

[4] NIF Project Control Manual Table of Contents, NIF-4165.

[5] NIF Quality Assurance Program Plan, NIF-618.

[6] RM Parsons NIF Title III Configuration Control Plan (Parsons Document 6/98), ref????

[7] Integrated Project Schedule.

[8] Preliminary Safety Analysis Report, NIF-LLNL-96-238, UCRL-ID-123759.

[9] Configuration Management Plan 96-070.

[10] Transition Plan. ???

[11] NIF Project Procedure 1.7, Project Change Control, NIF-3790

[12] Project Completion Criteria, NIF-1587-0A.

[13] NIF Work Breakdown Structure, NIF-5000220.

[14] NIF Construction Safety Program, UCRL-ID 125990 Rev 1, June 1997.

[15] NIF Conventional Facilities Construction Health and Safety Plan, NIF-4430.

[16] NIF Start-up/Operations and Special Equipment Construction Health and Safety Plan, NIF-2437.

[17] LLNL Health and Safety Manual, M-010.

[18] NIF Operations Procedures Plan, NIF-9004.

[19] NIF Software Configuration Management Plan, NIF-5476.

[20] NIF Project Procedure 10.1, Independent Assessments, ref????

[21] Project Procedure 8.3, Standard Content for Acceptance Test Procedures

[22] NIF Project Procedure 8.2., NIF-2052

[23] National Ignition Facility Project Execution Plan, UCRL-ID-126525.

[24] Project Procedure 6.4, Engineering Change Control, NIF-3132. 
[25] Project Procedure 1.3, Schedule Preparation, Statusing, and Revision, NIF-320 .

[26] Project Procedure 1.1, Preparation of Project Control Manual Procedures , NIF-95-469. 


\section{Appendix A - Glossary of Important Definitions}

Activation - synonymous with Start-up. The process that begins when all subsystems are completed for a given operational unit (such as a bundle), and effort is initiated to operate all subsystems together to make a laser system. Activation ends when a bundle is shown to meet its Operational Test Requirements. This marks the completion of the NIF Project's responsibility for the operational unit.

Acceptance test - a test conducted to determine whether a subsystem or component meets its requirements; if successful, work on that subsystem or component is complete except for integration into the next-higher-level system.

Bundle - a functional grouping of laser beams, 2 beams wide by 4 beams high. A bundle is the smallest unit that can be operated completely independently of all other similar units.

Component acceptance test - a test conducted to determine whether a component meets its requirements; if successful, work on that component is complete except for integration into the next-higherlevel system. Component acceptance tests are typically used as the final test before accepting items from vendors.

End of project - the formal end of construction of the NIF. This occurs when all project hardware is complete, installed, and has been acceptance tested. Operational tests will have been successfully completed on a portion of the overall system, as defined by the Project Completion Criteria. In general, all of Laser Bay 1 will have completed operational testing, and all of Laser Bay 2 will be installed and successfully through Subsystem Acceptance Tests.

ERP - Enterprise Resource Planning system. A computer software system that will be used for initiating and tracking procurement, maintaining hardware inventory, maintain bills of materials, etc.

Inspection - the process of comparing the physical configuration of a component to the design configuration and noting deviations.

Operational test - a test in which multiple subsystems are operated as an integrated unit. Typically, operational tests involve using NIF subsystems together to make an integrated laser system.

Start-up - see Activation.

Subsystem - a WBS level 3 entity. 
Subsystem acceptance test - a test conducted to determine whether a subsystem meets its requirements; if successful, work on that subsystem is complete and it is handed off to the NIF Start-up, Performance Testing, and Operations Group for activation. Typically subsystem acceptance tests will be conducted for a given operational unit, such as a bundle or cluster. The completion of subsystem acceptance tests for a given subsystem for all operational units marks the end of the Special Equipment Associate Project Engineer, System Engineer, or Lead Engineer's responsibility for that WBS element. 


\section{Appendix B - Summary of Title III Deliverables}

This appendix is a table that summarizes the Title III deliverables, which are described in detail in Section 4 (Table 1). In addition, the table indicates which of these deliverables is defined further by Appendix B.

\begin{tabular}{|c|c|}
\hline Project Deliverables & Appendix B Sample provided? \\
\hline - $\quad$ FSAR & no \\
\hline ORR for first bundle & no \\
\hline Project completion management review & no \\
\hline Project summary report & no \\
\hline \multicolumn{2}{|l|}{ Program Transition deliverables } \\
\hline procedures for turnover to operations & no - see [6] \\
\hline System Operations and Maintenance Plan & no \\
\hline OSPs & no - see [17] \\
\hline FSPs & no - see [17] \\
\hline $\begin{array}{l}\text { Subsystem operational description and draft } \\
\text { operating procedures }\end{array}$ & no - see [6] \\
\hline Final Operating Procedures & no - see [6] \\
\hline Maintenance plans and procedures & no - see [6] \\
\hline Final software operating manuals & no \\
\hline ICCS Software online help system & no \\
\hline Training development & no \\
\hline Management prestart review & no \\
\hline \multicolumn{2}{|l|}{ Configuration } \\
\hline as-built bills of material (by serial number) & B-1 \\
\hline As-built drawings, specifications, etc & B-2 \\
\hline Software Release Descriptions & no \\
\hline Configuration audit reports & no \\
\hline \multicolumn{2}{|l|}{ Design Basis information } \\
\hline - $\quad$ project files & no \\
\hline CAD models & no \\
\hline $\begin{array}{l}\text { Physics basis document, updated with actual test results } \\
\text { (1st bundle and LB1) }\end{array}$ & no \\
\hline Baseline Prop92 model (or equivalent) & no \\
\hline Title III Engineering Basis Book (includes other deliverables) & B-5 \\
\hline Fracture-Control Plans for brittle vacuum barriers & no \\
\hline supporting calculations & no \\
\hline \multicolumn{2}{|l|}{ Operational and pre-operational inspection and testing } \\
\hline - $\quad$ Final Subsystem Completion Checklists & B-3 \\
\hline Starup test history and data & no \\
\hline $\begin{array}{l}\text { part/ component history files by serial number } \\
\text { (where were they, and when) }\end{array}$ & B-4 \\
\hline System operational test information & no \\
\hline Subsystem acceptance test information & no \\
\hline Component inspection and test information & no \\
\hline QA plans and procedures & no - see [8] \\
\hline Software test results, with statistical analysis of RAM & no \\
\hline RAM database & no \\
\hline \multicolumn{2}{|l|}{ Title-III Implementation plans } \\
\hline $\begin{array}{l}\text { Updated schedules for procurement, fabrication, assembly, } \\
\text { installation and testing }\end{array}$ & no \\
\hline Final Transport/shipping/handling plan (by subsystem) & no-see [1] \\
\hline Final Assembly/installation/acceptance plan & no - see [1] \\
\hline \multicolumn{2}{|l|}{ Management deliverables } \\
\hline - $\quad$ CAPs (yearly) & no \\
\hline monthly / quarterly progress reports (written and oral) & no \\
\hline
\end{tabular}




\section{Appendix C - Sample Format and Content for Deliverables}

\section{Appendix C-1 - Sample format and content for As-Built Bills of Material}

This deliverable will be a standard table produced by the ERP System. It will be a complete indentured parts list showing actual part numbers and quantities used and listing serial numbers installed. A sample is shown below. In this sample, the design of an LRU was changed, and both new and old versions were used.

Bill of Material effective date: $\mathrm{mm} / \mathrm{dd} / \mathrm{yy}$

\begin{tabular}{|l|l|l|l|l|}
\hline $\begin{array}{l}\text { Indent } \\
\text { Level }\end{array}$ & Part No. & Qty & Part Name & Serial numbers \\
\hline 1 & AAA-123456 & 1 & NIF & \\
\hline 2 & AAA-123457 & 1 & LB\#1 Special Eq. & \\
\hline$\ldots$ & & & & \\
\hline 5 & AAA-234567 & 6 & LM1 LRU & $1,2,3,8,9,11$, \\
\hline 5 & AAA-345987 & 6 & LM1 LRU, mod 1 design & $23,24,25,26,31,32$ \\
\hline$\ldots$ & & & & \\
\hline 10 & N5305-1234 & 24 & Screw, M8x25, brass socket head & \\
\hline
\end{tabular}




\section{Appendix C-2 - Sample format and content for As-Built Dwgs, specifications, etc.}

This deliverable will be a standard table produced by the ERP System. It will be a complete indentured parts list showing actual part numbers used and listing all documents and drawings that describe each part. This will include drawings, specifications, assembly procedures, test results, etc. A sample is shown below.

Effective Date: $\mathrm{mm} / \mathrm{dd} / \mathrm{yy}$

\begin{tabular}{|l|l|ll|}
\hline $\begin{array}{l}\text { Indent } \\
\text { Level }\end{array}$ & Part No. & Described by & \\
\hline 1 & AAA-123456 & AAA-123456-ZZ & NIF Top Assembly dwg \\
\hline 2 & AAA-123457 & AAA-123457-0H & Laser Bay \#1 Assembly \\
\hline$\ldots$ & & & \\
\hline 5 & AAA-234567 & AAA-234567-0B & LM1 LRU Ass'y dwg \\
& & NIF-5001234-0C & LM1 LRU Ass' procedure \\
& & NIF-0022334-0A & LM1 LRU batch 1 Acceptance test data \\
& & NIF-0022445-0A & LM1 LRU batch 2 acceptance test data \\
\hline 5 & AAA-345987 & AAA-345987-0A & LM1 LRU, mod 1, Ass'y dwg \\
& & NIF-5001234-0D & LM1 LRU, mod 1, Ass'y procedure \\
& & NIF-5004567-0A & LM1 LRU, mod 1, new widget spec \\
& & NIF-0022556-0A & LM1 LRU batch 8 Acceptance test data \\
& & NIF-0022667-0A & LM1 LRU batch 9 acceptance test data \\
\hline$\ldots$ & & NIF-5000345-0A & NIF Quality Standard - Screws, metric, \\
\hline 10 & N5305-1234 & & brass, socket head \\
\hline
\end{tabular}




\section{Appendix C-3 - Sample format and content for Final Subsystem Completion Checklist}

The Subsystem Completion Checklist is intended to be a simple checklist of the items that must be handed off by the lead engineers and system engineers to the startup team before the subsystem is complete. The objective is to provide a vehicle for documenting the completion of each subsystem, as well as an index to other key pieces of documentation. The checklist will be different for each subsystem, but all will contain certain items in common. The following is a developmental draft of this checklist (all data is fictitious).

\section{Subsystem: WBS 1.3.1 Optical Pulse Generation}

Lead Engineer: Jim Davin

System Engineer: Doug Larson

\begin{tabular}{|c|c|c|c|}
\hline Item & Reference & $\begin{array}{l}\text { notes and comments on } \\
\text { status }\end{array}$ & $\begin{array}{l}\text { approved/restrictions } \\
\text { (signature/date) }\end{array}$ \\
\hline Turnover procedure & NIF-XYXYXY-0A & & clower $4 / 1 / 03$ \\
\hline OSPs & $\begin{array}{l}\text { OSP-XYXY } \\
\text { (NIF-XYXYXY) } \\
\text { OSP-1357 } \\
\text { (NIF-XYXYXY) }\end{array}$ & $\begin{array}{l}\text { for PAM testing in } \\
\text { PAMMA } \\
\text { for PABTS alignment } \\
\text { in Laser Bay }\end{array}$ & $\begin{array}{l}\text { clower } 4 / 1 / 03 \\
\text { clower } 4 / 1 / 03\end{array}$ \\
\hline $\begin{array}{l}\text { Operating } \\
\text { Procedures }\end{array}$ & NIF-XYXYXY-0A & $\begin{array}{l}\text { Procedures not } \\
\text { completed, but drafts } \\
\text { are sufficient in view } \\
\text { of schedule pressure }\end{array}$ & $\begin{array}{l}\text { clower } 4 / 1 / 03 \\
\text { conditional on } \\
\text { completion within } 60 \\
\text { days }\end{array}$ \\
\hline Maintenance Plans & $\begin{array}{l}\text { NIF-XYXYXY-0D } \\
\text { NIF-XYXYXY-0E }\end{array}$ & $\begin{array}{l}\text { for PAM } \\
\text { For PABTS }\end{array}$ & $\begin{array}{l}\text { clower } 4 / 1 / 03 \\
\text { clower } 4 / 1 / 03\end{array}$ \\
\hline $\begin{array}{l}\text { Maintenance } \\
\text { Procedures }\end{array}$ & NIF-XYXYXY-0D & $\begin{array}{l}\text { reference is } \\
\text { maintenance plan, } \\
\text { which refers to all } \\
\text { procedures }\end{array}$ & clower $4 / 1 / 03$ \\
\hline $\begin{array}{l}\text { as-built BOMs, } \\
\text { dwgs, specs }\end{array}$ & $\begin{array}{l}\text { in ERP, start with } \\
\text { Part AAA-XYXYXY }\end{array}$ & $\begin{array}{l}\text { BOM and all dwgs, } \\
\text { specs, and docs } \\
\text { released under CM } \\
\text { and up to date }\end{array}$ & clower $4 / 1 / 03$ \\
\hline CAD models & $\begin{array}{l}\text { in directory } \\
\text { NIF:sam:johnny:opg }\end{array}$ & $\begin{array}{l}\text { description of models } \\
\text { given to Sam }\end{array}$ & clower $4 / 1 / 03$ \\
\hline $\begin{array}{l}\text { Index to supporting } \\
\text { calcs }\end{array}$ & $\begin{array}{l}\text { All supporting calcs in } \\
\text { PDM as docs under } \\
\text { WBS } 1.3 .1\end{array}$ & & clower $4 / 1 / 03$ \\
\hline $\begin{array}{l}\text { Acceptance Test } \\
\text { Plans, procedures, } \\
\text { and test results }\end{array}$ & $\begin{array}{l}\text { NIF-XYXYXYX-0C } \\
\text { NIF-XYXYXYX-0B }\end{array}$ & $\begin{array}{l}\text { Acceptance Test Plan, } \\
\text { references all } \\
\text { procedures } \\
\text { Overall test result } \\
\text { summary document, } \\
\text { with references to } \\
\text { specific test results }\end{array}$ & $\begin{array}{l}\text { clower } 4 / 1 / 03 \\
\text { clower } 4 / 1 / 03\end{array}$ \\
\hline
\end{tabular}




\begin{tabular}{|l|l|l|l|}
\hline Item & Reference & $\begin{array}{l}\text { notes and comments on } \\
\text { status }\end{array}$ & $\begin{array}{l}\text { approved/restrictions } \\
\text { (signature/ date) }\end{array}$ \\
\hline $\begin{array}{l}\text { Inspection plans, } \\
\text { procedures, and test } \\
\text { results }\end{array}$ & NIF-XYXYXYX-0A & $\begin{array}{l}\text { Inspection Plan, } \\
\text { references all } \\
\text { procedures }\end{array}$ & clower 4/1/03 \\
& $\begin{array}{l}\text { NIF-XYXYXYX-0C } \\
\text { NIF-XYXYXYX-0B }\end{array}$ & $\begin{array}{l}\text { Overall inspection } \\
\text { record summary, with } \\
\text { references to specific } \\
\text { test results }\end{array}$ & clower 4/1/03 \\
\hline
\end{tabular}




\section{Appendix C-4 - Sample format and content for Part/Component History Files by Serial Number}

This deliverable will be a standard report produced by the ERP system on demand. It will show the entire history of any serialized part, including any processing or assembly internal to Lawrence Livermore National Laboratory, all installations, inspections, etc, that are done under a NIF work order.

Below is a sample of the type of information provided.

Part Number: AAA-123456 - Laser Amplifier Slab

Serial number: 2468

\begin{tabular}{|l|l|l|l|}
\hline Date & Action & PO/Work Order & Comments \\
\hline $4 / 1 / 02$ & received & PO 44444 & \\
\hline $4 / 2 / 02$ & transferred to OAB & WO 55555 & \\
\hline $4 / 3 / 02$ & cleaned & WO 55555 & \\
\hline $4 / 3 / 02$ & inspected & WO 55555 & see MDMS file abcdefg for data \\
\hline $4 / 3 / 02$ & assembled & WO 55555 & into LRU s/n 98765 \\
\hline $4 / 4 / 02$ & inspected & WO 55555 & passed \\
\hline $4 / 6 / 02$ & Installed & WO 87878 & location MA3/B31L \\
\hline $4 / 1 / 03$ & Removed & WO 98989 & \\
\hline $4 / 2 / 03$ & disassembled & WO 98989 & \\
\hline $4 / 2 / 03$ & inspected & WO 98989 & $\begin{array}{l}\text { see MDMS file abcdefg for data } \\
\text { ready for refurb }\end{array}$ \\
\hline $4 / 3 / 03$ & sent to refurb & PO 55555 & \\
\hline $5 / 3 / 03$ & received & PO 55555 & \\
\hline $5 / 2 / 03$ & transferred to OAB & WO 77777 & \\
\hline $5 / 3 / 03$ & cleaned & WO 77777 & \\
\hline $5 / 3 / 03$ & inspected & WO 77777 & see MDMS file abcdefg for data \\
\hline $5 / 3 / 03$ & assembled & WO 77777 & into LRU s/n 63245 \\
\hline $5 / 5 / 03$ & inspected & WO 77777 & passed \\
\hline $5 / 6 / 03$ & Installed & WO 34343 & location PA2/B24L \\
\hline $9 / 1 / 03$ & Removed & WO 56565 & \\
\hline $9 / 6 / 03$ & Installed & WO 56565 & location PA4/B12L \\
\hline
\end{tabular}




\section{Appendix C-5 - Title III Engineering Basis Book Outline}

The following table (next page) shows the outline of the Title III Engineering Basis Book in the left column.

The first 25 sections are the sections in the Title II Design Basis Book, as indicated by the checks in the second column. For Title III, most of these sections do not need to be resubmitted, because they are either obsolete (do not apply any longer), were completed by the design groups in Title II and new information is not relevant, or are replaced by a Title III deliverable. Some items, designated by " $U$ " in the third column, should be included in the Title III Book if they have been updated during Title III.

The Title III Engineering Basis book shall consist of any sections of the Title II Design Basis Book that have been updated, along with items 26 through 40. Items 26 to 40 either are new items (which had no counterpart in Title II design), or replace items in the Title II Design Basis Books. The right column lists the Title II item numbers that were replaced by each Title III deliverable.

Note that all items in the Title III Engineering Basis Book are listed separately as deliverables in the Title III Engineering Plan. The Title III book is simply a collection of that set of deliverables for each subsystem. 


\begin{tabular}{|c|c|c|c|c|}
\hline & $\begin{array}{l}\text { T-II } \\
\text { Deliver- } \\
\text { able? }\end{array}$ & $\begin{array}{c}\text { T-III } \\
\text { Deliver- } \\
\text { able? }\end{array}$ & $\begin{array}{c}\text { Title III } \\
\text { Deliverable } \\
\text { which replaces } \\
\text { listed Title II } \\
\text { Deliverable }\end{array}$ & $\begin{array}{c}\text { T-II Deliverable } \\
\text { which is replaced } \\
\text { by listed T-III } \\
\text { deliverable }\end{array}$ \\
\hline I TITLE II VIEWGRAPHS & $\sqrt{ }$ & & obsolete & \\
\hline \multicolumn{5}{|l|}{ II REQUIREMENTS } \\
\hline 1. Review for Compliance & $\sqrt{ }$ & & 36 & \\
\hline 2. $\mathrm{PC} / \mathrm{FR}, \mathrm{SDR}, \mathrm{SSDR}, \mathrm{SRS}$ & $\sqrt{ }$ & Update & & \\
\hline 3. ICDs & $\sqrt{ }$ & Update & & \\
\hline 4. Other Requirements & $\sqrt{ }$ & Update & & \\
\hline 5. Review of Physics Basis & $\sqrt{ }$ & & 36 & \\
\hline 6. Design Review Comments & $\sqrt{ }$ & & 36 & \\
\hline \multicolumn{5}{|l|}{ III DESIGN } \\
\hline 7. Product Data Structure & $\sqrt{ }$ & & 31 & \\
\hline 8. Drawing Package & $\sqrt{ }$ & & 32 & \\
\hline 9. Supporting Analysis & $\sqrt{ }$ & Update & & \\
\hline 10. Prototype & $\sqrt{ }$ & & 36 & \\
\hline 11. Software Models & $\sqrt{ }$ & & 27 & \\
\hline 12. RAM Budget/Analysis & $\sqrt{ }$ & & 33,38 & \\
\hline 13. FMEA Analysis & $\sqrt{ }$ & Update & 38 & \\
\hline 14. Q-Level Requirements & $\sqrt{ }$ & Update & & \\
\hline \multicolumn{5}{|l|}{ IV DESIGN IMPLEMENTATION PLANS } \\
\hline 15. Procurement Plan & $\sqrt{ }$ & & obsolete & \\
\hline 16. Transport/Shipping/Handling & $\sqrt{ }$ & Update & & \\
\hline 17. Assembly/Installation Plan & $\sqrt{ }$ & Update & & \\
\hline 18. ATPs Identified & $\sqrt{ }$ & & 36 & \\
\hline 19. Preliminary Master Training Plan & $\sqrt{ }$ & & completed & \\
\hline 20. Preliminary Operations Staffing & $\sqrt{ }$ & & completed & \\
\hline 21. Operations \& Maintenance Plans & $\sqrt{ }$ & & 27,28 & \\
\hline 22. Preliminary Software Manuals & $\sqrt{ }$ & & 29,30 & \\
\hline \multicolumn{5}{|l|}{ V PROCUREMENT READINESS } \\
\hline 23. Cost Estimate Reconciliation & $\sqrt{ }$ & & obsolete & \\
\hline 24. Schedules & $\sqrt{ }$ & & 39 & \\
\hline 25. Procurement Package & $\sqrt{ }$ & & obsolete & \\
\hline \multicolumn{5}{|l|}{ VI PROGRAM TRANSITION DELIVERABLES } \\
\hline $26 \quad 26$. OSPs & & $\sqrt{ }$ & & new \\
\hline $\begin{array}{l}\text { 27. Subsystem Operational Description and } \\
\text { draft operating procedures } \sqrt{ } \text { new, } 21,11\end{array}$ & & $\sqrt{ }$ & & new, 21, 11 \\
\hline 28. Maintenance plans and procedures $\sqrt{ } 21$ & & $\sqrt{ }$ & & 21 \\
\hline 29 . Final software operating manual $\sqrt{ } 22$ & & $\sqrt{ }$ & & 22 \\
\hline 30. ICCS software online help system $\sqrt{ } 22$ & & $\sqrt{ }$ & & 22 \\
\hline \multicolumn{5}{|l|}{ VIII CONFIGURATION } \\
\hline $\begin{array}{l}\text { 31. As-built bills of material (by } \\
\text { serial number) }\end{array}$ & & $\sqrt{ }$ & & 7 \\
\hline $\begin{array}{l}\text { 32. As-built drawings, } \\
\text { specifications, etc }\end{array}$ & & $\sqrt{ }$ & & 8 \\
\hline 33. Software Design Descriptions & & $\sqrt{ }$ & & 11 \\
\hline 34. Configuration audit reports & & $\sqrt{ }$ & & new \\
\hline \multicolumn{5}{|l|}{$\begin{array}{l}\text { VIII PRE-OPERATIONAL INSPECTION } \\
\text { AND TESTING }\end{array}$} \\
\hline $\begin{array}{l}\text { 35. Final Subsystem Completion } \\
\text { Checklists }\end{array}$ & & $\sqrt{ }$ & & new \\
\hline 36. Acceptance test information & & $\sqrt{ }$ & & new, $1,5,6,10,18$ \\
\hline 37. Inspection information & & $\sqrt{ }$ & & new \\
\hline $\begin{array}{l}\text { 38. Software test results, with } \\
\text { statistical analysis of RAM }\end{array}$ & & $\sqrt{ }$ & & new \\
\hline $\begin{array}{l}\text { 39. RAM database (demonstrating } \\
\text { that requirements will be met) }\end{array}$ & & $\sqrt{ }$ & & 12,13 \\
\hline IX TITLE-III IMPLEMENTATION PLANS & & & & \\
\hline $\begin{array}{l}\text { 40. Updated schedules for } \\
\text { procurement, fabrication, } \\
\text { assembly, installation and testing }\end{array}$ & & $\sqrt{ }$ & & 24 \\
\hline
\end{tabular}




\section{Appendix D - Revision Record}

\begin{tabular}{|l|c|l|l|}
\hline Revision & Date & \multicolumn{1}{|c|}{ By } & \multicolumn{1}{c|}{ Description } \\
\hline $0 \mathrm{~A}$ & $7 / 22 / 98$ & G. Deis & Initial Issue \\
\hline & & & \\
\hline
\end{tabular}

